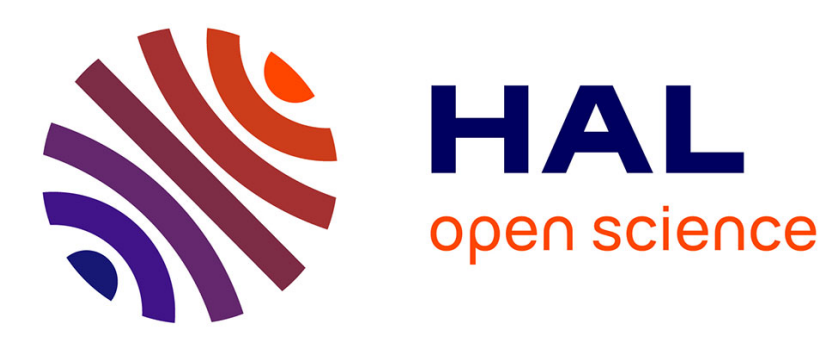

\title{
Whistler envelope solitons. I. Dynamics in inhomogeneous plasmas
}

\author{
C. Krafft, A.S. Volokitin
}

\section{To cite this version:}

C. Krafft, A.S. Volokitin. Whistler envelope solitons. I. Dynamics in inhomogeneous plasmas. Physics of Plasmas, 2018, 25 (10), pp.102301. 10.1063/1.5041055 . hal-01989769

\section{HAL Id: hal-01989769 \\ https://hal.sorbonne-universite.fr/hal-01989769}

Submitted on 22 Jan 2019

HAL is a multi-disciplinary open access archive for the deposit and dissemination of scientific research documents, whether they are published or not. The documents may come from teaching and research institutions in France or abroad, or from public or private research centers.
L'archive ouverte pluridisciplinaire HAL, est destinée au dépôt et à la diffusion de documents scientifiques de niveau recherche, publiés ou non, émanant des établissements d'enseignement et de recherche français ou étrangers, des laboratoires publics ou privés. 
Whistler envelope solitons. I. Dynamics in inhomogeneous plasmas

C. Krafft and A. Volokitin

A self-consistent Hamiltonian model based on equations describing the coupled dynamics of whistler and lower frequency waves in inhomogeneous plasmas is built. On this basis, different aspects of whistler turbulence are studied, concerning mainly the development of modulational instabilities and the dynamics of envelope solitons in irregular plasmas. Numerical simulations based on the model show that modulational instability can lead to the generation of a beating of stable nonlinear whistlers propagating with a speed near the group velocity. The whistler envelope soliton is determined analytically and its propagation in plasmas presenting random density fluctuations and weakly irregular density structures of different scales and amplitudes is studied, showing that the envelope is very weakly affected by these inhomogeneities, whereas the wavelengths and the amplitudes of the phase oscillations strongly vary. Moreover, simulations show for the first time that two whistler solitons moving with different but close velocities and colliding one with the other remain unchanged after this collision, independently of their initial amplitudes and velocities. Finally we study the dynamics of sonic whistler envelope solitons and show that the propagation of their lower frequency perturbation is governed by a KdV-type equation. 


\section{INTRODUCTION}

The emission of whistlers excited by electron beams in the presence of very low frequency acoustic-type waves and plasma inhomogeneities has been observed in several space experiments. For example, low frequency electromagnetic waves identified as whistlers were detected in the inhomogeneous solar wind plasma in association with Langmuir and ion acoustic waves ${ }^{1,2}$. The Wind spacecraft observed parallel propagating whistlers and Langmuir waves within a coronal mass ejection ${ }^{3}$, which were very good time-correlated with a loss-cone electron distribution within a magnetic cloud. Whistler waves propagating along magnetic loops during solar flares and generated by loss-cone distributions of relativistic electrons have been shown to be responsible for the generation of fiber bursts in the solar radio radiation ${ }^{4}$. Normal cyclotron mechanisms driven by temperature anisotropy have been identified $^{5}$ to be the cause of lion roars, which are intense and narrowband whistlers of low frequency, that are one of the characteristic features of the magnetosheath. Moreover, this mechanism is responsible for whistler emission upstream of the Earth's bowshock and at interplanetary shocks ${ }^{6}$.

Bursts of narrowband and short-living whistlers have been frequently observed by spacecraft ${ }^{7}$; most of these waves propagate in the electron foreshock quasi-parallel to the ambient magnetic field, and are likely produced by electron fluxes through cyclotron resonances and temperature anisotropy; they are more rarely observed in the solar wind, where they are believed to propagate along the field lines and originate from distant sources (such as interplanetary shocks ${ }^{8}$ ) or halo electrons presenting temperature anisotropy and carrying heat flux ${ }^{9}$. The spacecraft Cluster observed in the solar wind narrow band coherent fluctuations propagating quasi-parallel to the magnetic field, interpreted as whistler waves ${ }^{10}$. The specific problem of interaction between narrow whistler waves packets and warm electron beams (i.e. with finite perpendicular and parallel temperatures) is particularly interesting. Indeed, as whistlers excited by such beams present in many cases positive linear growth rates only in narrow spectral bands ${ }^{11}$ (see also below), one can suppose that, even in the quasilinear and nonlinear stages of the evolution, the waves with resonant velocities in the corresponding narrow velocity domain should play the most significant role. In this case a broadband packet is not necessary for producing an efficient interaction with electron fluxes and a subsequent significant radiation. Such whistler waves, for example, can be generated due to some natural physical processes, as evidenced by the space observations mentioned just above, or be emitted in the magnetosphere by ground transmitters ${ }^{12}$.

The coupling of large amplitude whistlers as those observed in space plasmas ${ }^{13}$ with lower frequency waves as ion or electron acoustic waves can lead to various phenomena developing in the nonlinear stage of the evolution, in presence or absence of resonant particles, as ponderomotive effects, modulational instabilities, formation of stable structures as solitons or localized wave packets, etc. The satellite $O g o 5$ observed isolated discrete whistler packets in the solar wind ${ }^{14}$. Observations by spacecraft revealed the existence of localized electric 
fields and density structures in the high and low frequency ranges. Indeed, the satellite Freja observed right-polarized wave packets accompanied by density cavities with depths of the order of $1 \%$ of the background plasma, identified by the authors as envelope whistler solitary waves ${ }^{15}$. The space mission Cluster detected whistler emissions near the plasmapause, correlated with density fluctuations aligned along the ambient magnetic field ${ }^{16}$, which are modulated structures associated with the density perturbations. Moreover, the satellite Fast observed envelope solitary electron-acoustic waves propagating along the magnetic field lines in auroral density cavities, in regions where two electrons populations, hot and cold, exist ${ }^{17}$. Finally, some authors report the observations of envelope soliton fine structures observed in solar radio metric-wave emissions occurring during solar radio bursts ${ }^{18}$.

In order to explain such observations, we present hereafter a self-consistent Hamiltonian model based on equations describing the coupled dynamics of electromagnetic whistlers and lower frequency waves ${ }^{19,20}$ in randomly inhomogeneous plasmas. This model is used, owing to an associated numerical code, to study the characteristic features of nonlinear structures as solitons and physical effects as modulational instabilities, which can rise in space plasmas, as mentioned above. The present paper extends and generalizes the existing studies ${ }^{21-23}$, by enlarging the domain of parameters' validity (e.g. by considering the full range of whistler frequencies, and not only the limiting case of very low frequencies) and by providing detailed numerical simulations' results on different aspects of whistler turbulence in inhomogeneous plasmas. In particular and to our knowledge, the dynamics of the collision between whistler envelope solitons is presented for the first time. Concerning modulational instabilities, we also show how they can generate beatings between stable nonlinear structures. Moreover the whistler soliton propagation is studied in plasmas presenting external preexisting irregularities in the form of random fluctuations, which are particularly important as they can strongly influence on the nonlinear effects at work, as it was evidenced recently for the case of Langmuir turbulence in the solar wind ${ }^{24-27}$. Note that the model provides useful invariants which allow to control the numerical simulations; moreover it can be completed by adding populations of resonant particles interacting with the solitons, as discussed in the companion paper ${ }^{32}$.

\section{INTERACTION OF WHISTLERS WITH LOWER FREQUENCY WAVES : THEORETICAL MODEL}

In a magnetized plasma, whistlers can couple nonlinearly to lower frequency waves, due to the ponderomotive force and the interactions between their fields and the plasma fluid density's, velocity's and magnetic field's perturbations. In this frame, specific physical effects can rise and influence strongly on the dynamics of the whistlers.

Let us consider the case of whistler wave packets propagating parallel to the ambient 
magnetic field $\mathbf{B}_{0}$; their magnetic field is assumed to be of the form

$$
\mathbf{B}=\operatorname{Re}\left(B(z, t) e^{-i \omega_{0} t+i k_{0} z}(\mathbf{x}+i \mathbf{y})\right)
$$

where $B=B_{x}-i B_{y}$ is the slowly varying (with space and time) envelope of the right circularly polarized wave; $\mathbf{x}$ and $\mathbf{y}$ are the unitary vectors along the axes perpendicular to the direction $\mathbf{z}$ of the wave propagation and the ambient magnetic field $\mathbf{B}_{0} ; B_{x}$ and $B_{y}$ are the coordinates of $\mathbf{B}$ along $\mathbf{x}$ and $\mathbf{y} ; \omega_{0}$ and $k_{0}$ are the central frequency and wavenumber of the whistler packet. The dispersion relation is given by

$$
\mathcal{D}\left(\omega_{0}, k_{0}\right)=k_{0}^{2} c^{2}-\omega_{0}^{2}\left(\varepsilon_{\perp}-q\right)=k_{0}^{2} c^{2}-\omega_{0}^{2}+\frac{\omega_{p}^{2} \omega_{0}}{\omega_{0}-\omega_{c}}=0
$$

with the elements of the cold dielectric tensor

$$
\varepsilon_{\perp}=1-\frac{\omega_{p}^{2}}{\omega_{0}^{2}-\omega_{c}^{2}}, \quad q=\frac{\omega_{c} \omega_{p}^{2}}{\omega_{0}\left(\omega_{0}^{2}-\omega_{c}^{2}\right)},
$$

where $\omega_{p}$ and $\omega_{c}$ are the electron plasma and cyclotron frequencies, respectively. Note that, contrary to previous studies ${ }^{22}$ which considered only waves with $\omega_{0} \ll \omega_{c}$, our model takes into account the full range of whistler frequencies, i.e. $\omega_{l h}<\omega_{0}<\omega_{c}$, where $\omega_{l h}$ is the lower hybrid frequency. Combining the Maxwell equations we obtain that

$$
\frac{1}{c^{2}} \frac{\partial^{2} \mathbf{B}}{\partial t^{2}}-\nabla^{2} \mathbf{B}=\frac{4 \pi}{c} \nabla \times \mathbf{j}
$$

where $\mathbf{j}$ is the current density involving the external currents $\mathbf{j}_{\text {ext }}$ (which can be carried by an electron beam injected in the plasma, for example). Using the Ohm's law for the linear current density, and taking into account the slow space and time variations of the magnetic field envelope $B$, we obtain that $\mathcal{D}(\omega, k) B \simeq 0$ when $\mathbf{j}_{\text {ext }}=0$ (for more details see the Appendix A). Performing a Taylor development of $\mathcal{D}(\omega, k)$ around $\left(\omega_{0}, k_{0}\right)$ up to order two (parabolic approximation) and keeping only the main terms, we can write

$$
\begin{aligned}
\mathcal{D}(\omega, k) \simeq i\left(\frac{\partial \mathcal{D}}{\partial \omega}\right)_{0} & \frac{\partial}{\partial t}-i\left(\frac{\partial \mathcal{D}}{\partial k}\right)_{0} \frac{\partial}{\partial z}-\frac{1}{2}\left(\left(\frac{\partial^{2} \mathcal{D}}{\partial \omega^{2}}\right)_{0} \frac{\partial^{2}}{\partial t^{2}}-2\left(\frac{\partial^{2} \mathcal{D}}{\partial k \partial \omega}\right)_{0} \frac{\partial^{2}}{\partial z \partial t}+\left(\frac{\partial^{2} \mathcal{D}}{\partial k^{2}}\right)_{0} \frac{\partial^{2}}{\partial z^{2}}\right) \\
& +\rho\left(\frac{\partial \mathcal{D}}{\partial \rho}\right)_{0}+\delta v_{e}\left(\frac{\partial \mathcal{D}}{\partial \delta v_{e}}\right)_{0}+\frac{\delta B_{z}}{B_{0}}\left(\frac{\partial \mathcal{D}}{\partial\left(\delta B_{z} / B_{0}\right)}\right)_{0},
\end{aligned}
$$

where $\rho=\delta n_{e} / n_{0}$ and $\delta v_{e}$ are the perturbations of the slowly varying electron fluid density and velocity; $\delta B_{z}$ is the slowly varying magnetic field perturbation along $\mathbf{z} ; n_{0}$ is the unperturbed plasma density at equilibrium. Then the equation of evolution of the wave magnetic field envelope is obtained in the following form (see also the Appendix A)

$$
i \frac{\partial B}{\partial t}+i v_{g 0} \frac{\partial B}{\partial z}+\frac{v_{g 0}^{\prime}}{2} \frac{\partial^{2} B}{\partial z^{2}}=-\frac{\omega_{0} \omega_{p}^{2}}{\mathcal{D}_{0}^{\prime}\left(\omega_{0}-\omega_{c}\right)}\left(\rho+\frac{\omega_{c}}{\omega_{0}-\omega_{c}}\left(\frac{k_{0}}{\omega_{0}} \delta v_{e}+\frac{\delta B_{z}}{B_{0}}\right)\right) B
$$


with

$$
\mathcal{D}_{0}^{\prime}=\left(\frac{\partial \mathcal{D}}{\partial \omega}\right)_{0}=-\left(2 \omega_{0}+\frac{\omega_{p}^{2} \omega_{c}}{\left(\omega_{c}-\omega_{0}\right)^{2}}\right) .
$$

The right hand side of (6) consists of three nonlinear terms representing the coupling between the whistler field and the lower frequency perturbations of density, velocity and ambient magnetic field. Note that $\mathcal{D}_{0}^{\prime}=-\omega_{0}^{2} \varepsilon_{0}^{\prime}$, where $\varepsilon_{0}^{\prime}$ is the derivative of the dielectric constant at $\left(\omega_{0}, k_{0}\right)$. The group velocity and its derivative are given by $v_{g 0}=(d \omega / d k)_{0}=$ $-(\partial \mathcal{D} / \partial k)_{0} /(\partial \mathcal{D} / \partial \omega)_{0}=-2 c^{2} k_{0} / \mathcal{D}_{0}^{\prime}$ and

$$
v_{g 0}^{\prime}=\left(\frac{d^{2} \omega}{d k^{2}}\right)_{0}=\frac{v_{g 0}}{k_{0}}\left(1-\frac{v_{g 0}^{2}}{c^{2}}\left(1+\frac{\omega_{p}^{2} \omega_{c}}{\left(\omega_{c}-\omega_{0}\right)^{3}}\right)\right) .
$$

Below the term with $\delta B_{z}$ in (6) will be omitted as the magnetic field $\mathbf{B}_{0}$ is considered here to be constant. The evolution of the lower frequency oscillations of the electron density $\delta n_{e}$ and velocity $\delta v_{e}$ can be obtained by using the momentum conservation equation in the steady state

$$
\frac{\partial}{\partial t} \delta v_{e}+\delta v_{e} \frac{\partial}{\partial z} \delta v_{e}=\frac{e}{m_{e}} \frac{\partial}{\partial z} \delta \varphi-\frac{T_{e}}{n_{e} m_{e}} \frac{\partial}{\partial z} \delta n_{e}+\frac{F_{p z}}{m_{e}} \simeq 0
$$

where $\delta \varphi$ is the low frequency potential; $T_{e}, m_{e}$ and $-e<0$ are the electron plasma temperature, mass and charge, respectively. The ponderomotive force $F_{p z}$ of whistlers acting on the particles is (see also the calculations of the non stationary part of the force in the Appendix B)

$$
F_{p z}=-\frac{\omega_{p}^{2}}{\omega_{0}\left(\omega_{0}-\omega_{c}\right)}\left(\frac{\partial}{\partial z}-\frac{\omega_{c}}{\left(\omega_{0}-\omega_{c}\right)} \frac{k_{0}}{\omega_{0}} \frac{\partial}{\partial t}\right) \frac{|E|^{2}}{16 \pi n_{0}}
$$

where $E=\left(\omega_{0} / c k_{0}\right) B$; the expression (9) is similar to that obtained in another way ${ }^{28,29}$. The ion dynamics is described by

$$
\begin{gathered}
\frac{\partial}{\partial t} \delta n_{i}+\frac{\partial}{\partial z}\left(n_{i} \delta v_{i}\right)=0 \\
\frac{\partial}{\partial t} \delta v_{i}+\delta v_{i} \frac{\partial}{\partial z} \delta v_{i}=-\frac{e}{m_{i}} \frac{\partial}{\partial z} \delta \varphi-\frac{T_{i}}{n_{i} m_{i}} \frac{\partial}{\partial z} \delta n_{i}
\end{gathered}
$$

where $\delta n_{i}\left(n_{i}=n_{0}+\delta n_{i}\right)$ and $\delta v_{i}$ are the proton density and velocity perturbations; $T_{i}$ and $m_{i}$ are the ion plasma temperature and mass. In the case of quasineutral low frequency oscillations $\left(\rho=\delta n_{e} / n_{0} \simeq \delta n_{i} / n_{0}\right.$ and consequently, due to charge conservation, $V=\delta v_{e} \simeq$ $\left.\delta v_{i}\right)$, one can get the low frequency equations in the form

$$
\begin{gathered}
\frac{\partial}{\partial t}\left(V-\frac{\omega_{p}^{2}}{\left(\omega_{0}-\omega_{c}\right)^{2}} \frac{\omega_{c}}{k_{0}} \frac{|B|^{2}}{16 \pi n_{0} m_{i} c^{2}}\right)+\frac{\partial}{\partial z}\left(c_{s}^{2} \rho+\frac{\omega_{p}^{2} \omega_{0}}{k_{0}^{2}\left(\omega_{0}-\omega_{c}\right)} \frac{|B|^{2}}{16 \pi n_{0} m_{i} c^{2}}\right) \simeq 0, \\
\frac{\partial \rho}{\partial t}+\frac{\partial V}{\partial z} \simeq 0,
\end{gathered}
$$

where the hydrodynamic nonlinear terms have been neglected and the ponderomotive terms have been expressed as a function of the magnetic field enevelope $B ; c_{s}$ is the ion sound 
velocity. Defining $|\mathcal{B}|^{2}=|B|^{2} / 16 \pi n_{0} m_{i} c^{2}$ and introducing the hydrodynamic flux $\Psi$ in the form

$$
\frac{\partial \Psi}{\partial z}=V-\frac{\omega_{c} \omega_{p}^{2}}{k_{0}\left(\omega_{c}-\omega_{0}\right)^{2}}|\mathcal{B}|^{2}
$$

the equations (12)-(13) can be written as

$$
\frac{\partial \Psi}{\partial t}+c_{s}^{2} \rho+\frac{\omega_{p}^{2} \omega_{0}}{k_{0}^{2}\left(\omega_{0}-\omega_{c}\right)}|\mathcal{B}|^{2}=0
$$

and

$$
\frac{\partial^{2} \Psi}{\partial z^{2}}+\frac{\partial \rho}{\partial t}+\frac{\omega_{p}^{2} \omega_{c}}{k_{0}\left(\omega_{0}-\omega_{c}\right)^{2}} \frac{\partial|\mathcal{B}|^{2}}{\partial z}=0
$$

Adding the equation for the whistler envelope in the following form $\left(\delta B_{z}=0\right)$

$$
i \frac{\partial \mathcal{B}}{\partial t}+i v_{g 0} \frac{\partial \mathcal{B}}{\partial z}+\frac{v_{g 0}^{\prime}}{2} \frac{\partial^{2} \mathcal{B}}{\partial z^{2}}=-\frac{\omega_{p}^{2}}{\left(\omega_{0}-\omega_{c}\right) \mathcal{D}_{0}^{\prime}}\left(\omega_{0} \rho \mathcal{B}+\frac{k_{0} \omega_{c}}{\left(\omega_{0}-\omega_{c}\right)} \frac{\partial \Psi}{\partial z} \mathcal{B}+\frac{\omega_{c}^{2} \omega_{p}^{2}|\mathcal{B}|^{2} \mathcal{B}}{\left(\omega_{0}-\omega_{c}\right)^{3}}\right)
$$

we get a system of three differential equations whose Hamiltonian can be expressed as (see also the Appendix A)

$$
\begin{aligned}
\mathcal{H}=\int \frac{d z}{L} H=\int \frac{d z}{L} \times \\
{\left[\frac{i v_{g 0}}{2}\left(\mathcal{B} \frac{\partial \mathcal{B}^{*}}{\partial z}-\mathcal{B}^{*} \frac{\partial \mathcal{B}}{\partial z}\right)+\frac{v_{g 0}^{\prime}}{2}\left|\frac{\partial \mathcal{B}}{\partial z}\right|^{2}+\frac{\omega_{p}^{2} \omega_{0} \rho|\mathcal{B}|^{2}}{\mathcal{D}_{0}^{\prime}\left(\omega_{c}-\omega_{0}\right)}-\frac{k_{0}^{2}}{2 \mathcal{D}_{0}^{\prime}}\left(V^{2}+c_{s}^{2} \rho^{2}\right)\right] }
\end{aligned}
$$

where $L$ is the size of the system and $V$ has been expressed using (14). The three first terms of (18) represent the energy of the whistler waves, the fourth one the energy of interaction between the whistlers and the lower frequency waves, and the two last the energy of the lower frequency waves. Another invariant, i.e. the momentum $\mathcal{P}$ of the system, can be deduced from (12)-(13)-(17)

$$
\mathcal{P}=i \int\left(\mathcal{B} \frac{\partial \mathcal{B}^{*}}{\partial z}-\mathcal{B}^{*} \frac{\partial \mathcal{B}}{\partial z}\right) \frac{d z}{L}+\frac{2 k_{0}^{2}}{\mathcal{D}_{0}^{\prime}} \int \rho V \frac{d z}{L}-\frac{2 \omega_{p}^{2}}{\mathcal{D}_{0}^{\prime}} \frac{k_{0} \omega_{c}}{\left(\omega_{c}-\omega_{0}\right)^{2}} \int \rho|\mathcal{B}|^{2} \frac{d z}{L}
$$

The last invariant is the number of quanta

$$
\mathcal{H}_{0}=\int \omega_{0}|\mathcal{B}|^{2} \frac{d z}{L}
$$

which can also be added to (18). The two couples of canonical variables are $\left(\mathcal{B}, \mathcal{B}^{*}\right)=$ $\left(B, B^{*}\right) / \sqrt{16 \pi n_{0} m_{i} c^{2}}$ and $(\Psi, \Pi)=\left(\Psi,-k_{0}^{2} \rho / \mathcal{D}_{0}^{\prime}\right)$. One can check that the corresponding Hamilton equations are given by

$$
\frac{\partial \Pi}{\partial t}=\frac{\delta H}{\delta \Psi}, \quad \frac{\partial \Psi}{\partial t}=-\frac{\delta H}{\delta \Pi}, \quad \frac{\partial \mathcal{B}}{\partial t}=-i \frac{\delta H}{\delta \mathcal{B}^{*}},
$$


where $\delta$ is the functional derivative. This Hamiltonian system can used to study the interactions of whistlers with ion sound waves, for example. Note that, due to (5), the model is valid for the case of narrowband coherent whistlers propagating quasi-parallel to the magnetic field lines. Such whistlers can be generated naturally in space or artificially in laboratory experiments, using pump waves or particles' fluxes, as discussed in the companion paper of this $\operatorname{article}^{32}$.

Our purpose is now to solve the system formed by (18) and (21) (or (6), (12) and (13)) in order to understand the dynamics of a whistler packet interacting with ion sound waves. A numerical code has been built which is based on the normalized model's equations, which are solved using pseudo-spectral methods, discretization schemes and Fast Fourier Transforms (see the Appendix C for more details). Packets of 1024-4096 waves of wavenumbers $k_{0} \pm k\left(0<k \ll k_{0}\right)$ and frequencies $\omega_{k_{0} \pm k}$ are used, which present initially narrowband spectra peaked at the central wavenumber $k_{0}$ at frequency $\omega_{0}$. The one-dimensional simulation box extends typically over a normalized distance $L \omega_{c} / c \simeq 10^{3}-10^{5}$. External plasma inhomogeneities can be introduced as initial perturbations. The accuracy of the calculations is controlled owing to the model's invariants, i.e. the number of quanta (20), the system's energy (18) and momentum (19).

\section{MODULATIONAL INSTABILITY}

As shown in previous studies ${ }^{21}$, whistler waves propagating along magnetic field lines can be modulationally unstable with respect to perturbations of the lower frequency range. On the basis of the above presented Hamiltonian model we will first determine the growth rate of such instabilities and then perform numerical simulations.

Let us express the magnetic field envelope as $B=B_{k_{0}}+B_{k_{0}+\kappa} e^{i \kappa z-i \Omega t}+B_{k_{0}-\kappa} e^{-i \kappa z+i \Omega t}$, where the amplitude of the Fourier component $B_{k_{0}}$ corresponding to the central wavenumber $k_{0}$ is large compared to the amplitudes of the other components $B_{k_{0} \mp \kappa}$. So the density and velocity perturbations satisfy $\rho=\rho_{\kappa} e^{i \kappa z-i \Omega t}+\rho_{-\kappa} e^{-i \kappa z+i \Omega t}$ and $V=V_{\kappa} e^{i \kappa z-i \Omega t}+V_{-\kappa} e^{-i \kappa z+i \Omega t}$. From (13) one can deduce that $\Omega \rho_{\kappa}=\kappa V_{\kappa}$. Moreover, in the Fourier space the equations (6) and (12) lead respectively to the relations

$$
\left( \pm \Omega \mp \kappa v_{g 0}-\frac{\kappa^{2} v_{g 0}^{\prime}}{2}\right) B_{k_{0} \pm \kappa}=-\frac{\omega_{0} \omega_{p}^{2}}{\mathcal{D}_{0}^{\prime}\left(\omega_{0}-\omega_{c}\right)}\left(\frac{\kappa}{\Omega}+\frac{\omega_{c}}{\omega_{0}-\omega_{c}} \frac{k_{0}}{\omega_{0}}\right) V_{ \pm \kappa} B_{k_{0}}
$$

and

$\Omega\left(V_{\kappa}-\frac{\omega_{p}^{2}}{\left(\omega_{0}-\omega_{c}\right)^{2}} \frac{\omega_{c}}{k_{0}} \frac{B_{k_{0}}^{*} B_{k_{0}+\kappa}+B_{k_{0}} B_{k_{0}-\kappa}^{*}}{16 \pi n_{0} m_{i} c^{2}}\right)=\kappa\left(c_{s}^{2} \rho_{\kappa}+\frac{\omega_{p}^{2} \omega_{0}}{k_{0}^{2}\left(\omega_{0}-\omega_{c}\right)} \frac{B_{k_{0}}^{*} B_{k_{0}+\kappa}+B_{k_{0}} B_{k_{0}-\kappa}^{*}}{16 \pi n_{0} m_{i} c^{2}}\right)$,

where the asterisk indicates the complex conjugate. Combining the equations (22) and (23) we get

$$
\left(\Omega^{2}-\kappa^{2} c_{s}^{2}\right)\left(\Omega-\kappa v_{g 0}-\frac{\kappa^{2} v_{g 0}^{\prime}}{2}\right)\left(\Omega-\kappa v_{g 0}+\frac{\kappa^{2} v_{g 0}^{\prime}}{2}\right)
$$




$$
=-\frac{\omega_{0}^{2} \omega_{p}^{4} v_{g 0}^{\prime} \kappa^{2}}{\mathcal{D}_{0}^{\prime}\left(\omega_{0}-\omega_{c}\right)^{2}}\left(\frac{\kappa}{k_{0}}+\frac{\omega_{c}}{\left(\omega_{0}-\omega_{c}\right)} \frac{\Omega}{\omega_{0}}\right)^{2} \frac{\left|B_{k_{0}}\right|^{2}}{16 \pi n_{0} m_{i} c^{2}}
$$

or also

$$
\Omega^{2}-\kappa^{2} c_{s}^{2}=\Gamma^{3} \frac{\kappa^{2} v_{g 0}^{\prime}\left(\frac{\kappa}{k_{0}}+\frac{\omega_{c}}{\left(\omega_{0}-\omega_{c}\right)} \frac{\Omega}{\omega_{0}}\right)^{2}}{\left(\Omega-\kappa v_{g 0}\right)^{2}-\left(\kappa^{2} v_{g 0}^{\prime} / 2\right)^{2}}
$$

where

$$
\Gamma^{3}=\frac{\omega_{0}^{2} \omega_{p}^{2}}{c k_{0}} \frac{\omega_{c}^{2}}{\left(\omega_{0}-\omega_{c}\right)^{2}} \frac{v_{g 0}}{8 c} \frac{\omega_{p}^{2}}{\omega_{c}^{2}}\left(\frac{v_{A}}{c}\right)^{2} \frac{\left|B_{k_{0}}\right|^{2}}{B_{0}^{2}}>0,
$$

$v_{A}$ being the Alfven velocity; note that $v_{g 0}$ has the same sign as $k_{0}$. Then, searching solutions of (25) for small $\kappa$ that satisfy $\Omega \simeq \kappa v_{g 0}+i \gamma$ with $\left|\kappa^{2} v_{g 0}^{\prime} / 2\right| \ll\left|\kappa v_{g 0}\right|$, we get

$$
\gamma^{2} \simeq \Gamma^{3}\left(\frac{v_{g 0}^{\prime}}{c_{s}^{2}-v_{g 0}^{2}}\right)\left(\frac{\kappa}{k_{0}}\right)^{2}\left(1-\frac{\omega_{c}}{\left(\omega_{c}-\omega_{0}\right)} \frac{k_{0} v_{g 0}}{\omega_{0}}\right)^{2}
$$

which shows that modulational instability can exist for supersonic $\left(v_{g 0}>c_{s}\right)$ or subsonic $\left(v_{g 0}<c_{s}\right)$ whistlers only if $v_{g 0}^{\prime}<0$ or $v_{g 0}^{\prime}>0$, respectively. Note that $\gamma$ is proportional to $\left|B_{k_{0}} / B_{0}\right|$ through $\Gamma^{3}$. For the strongly supersonic case when $\Omega^{2} \gg c_{s}^{2} \kappa^{2}$ (i.e. $v_{g 0} \gg c_{s}$ ) we get the growth rate

$$
\gamma=\Gamma^{3 / 2} \frac{\left|v_{g 0}^{\prime}\right|^{1 / 2}}{v_{g 0}} \frac{\kappa}{k_{0}}\left|1-\frac{\omega_{c}}{\left(\omega_{c}-\omega_{0}\right)} \frac{k_{0} v_{g 0}}{\omega_{0}}\right| .
$$

If $v_{g 0} \simeq c_{s}$ (sonic case), the growth rate given by (27) presents a singularity. However the relations (??) allow to write in this case that $\kappa\left(c_{s}-v_{g 0}\right) \simeq i \gamma$ so that we get from (27) that

$$
\gamma \simeq i^{1 / 3} \kappa \Gamma\left(\frac{v_{g 0}^{\prime}}{2 k_{0}^{2} v_{g 0}}\right)^{1 / 3}\left(1-\frac{\omega_{c}}{\left(\omega_{c}-\omega_{0}\right)} \frac{k_{0} v_{g 0}}{\omega_{0}}\right)^{2 / 3}
$$

with $i^{1 / 3}=0.866-0.5 i$. Let us solve numerically the equation (25) and show in Fig. 1 the variations of the growth rates $\operatorname{Im}\left(\Omega / \omega_{0}\right)$ as a function of the normalized wavenumbers $\kappa / k_{0}$ for three examples of space plasma parameters, i.e. for a very low frequency subsonic whistler in the solar wind (Fig. 1, upper panel), a supersonic whistler and a sonic whistler of high frequency $\left(\omega_{0} \simeq \omega_{c}\right)$ in the Earth magnetosphere (Fig. 1, lower panels). As expected by the nature of the modulational instability, the calculated growth rates are rather weak, even if at large $B / B_{0} \sim 0.3$ they can reach a significative value around $10^{-3}$ (see Fig. 1, upper panel) and can thus a priori compete with other nonlinear phenomena, as the three-wave resonant interaction process where a whistler decays into a backscattered whistler and an ion sound wave, for example. However, according to Ref. 28 where the authors considered the different classes of parametric instabilities for whistlers propagating along magnetic field lines in a dense plasma, these instabilities develop in different ranges of $k$. Moreover, in the case of narrowband whistlers, different effects should not compete one with another in narrow $k$ ranges, for the same physical parameters. 

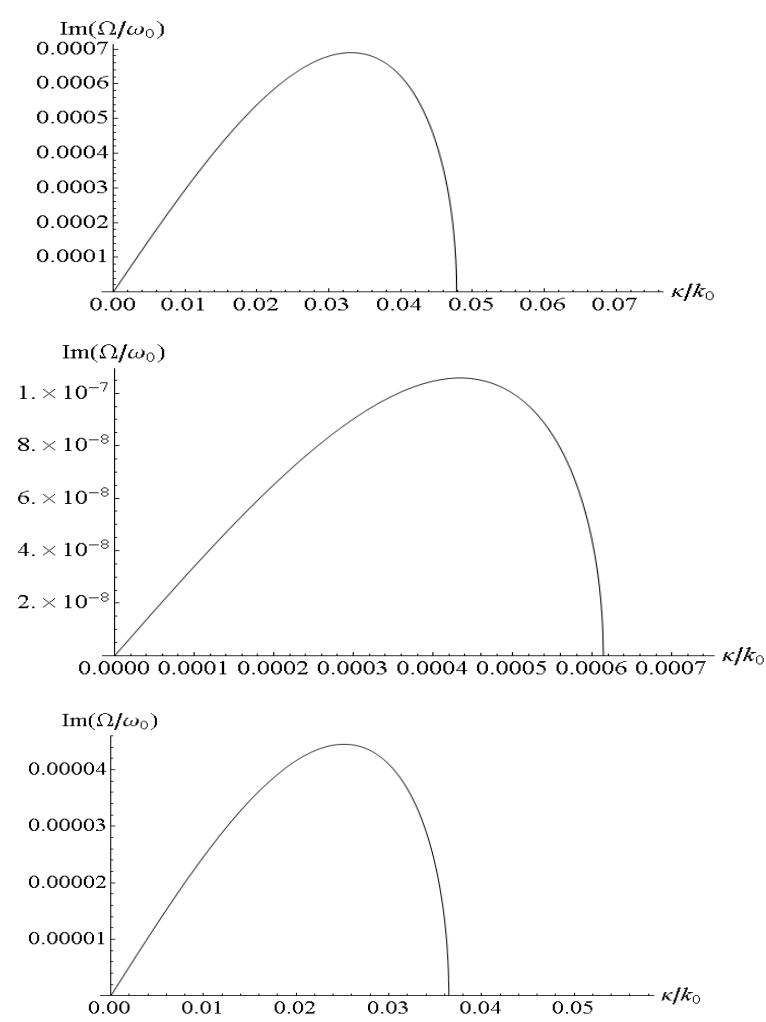

FIG. 1. Growth rate $\operatorname{Im}\left(\Omega / \omega_{0}\right)$ of the modulational instability as a function of the normalized wavenumber $\kappa / k_{0}$. (Upper panel) : Subsonic case $\left(v_{g 0}<c_{s}\right)$, for parameters typical of the solar wind : $\omega_{p} / \omega_{c}=110, \omega_{0} / \omega_{c}=0.0013, c k_{0} / \omega_{c}=4, c_{s}=0.0008, B / B_{0}=0.28, v_{g 0}=0.00065$, $v_{g 0}^{\prime}>0$. (Middle panel) : Supersonic case $\left(v_{g 0}>c_{s}\right)$, for parameters typical of the magnetosphere : $\omega_{p} / \omega_{c}=3, \omega_{0} / \omega_{c}=0.59, c k_{0} / \omega_{c}=3.7, B / B_{0}=0.017, c_{s}=0.002, v_{g 0}=0.13, v_{g 0}^{\prime}<0$. (Lower panel): Sonic case $\left(v_{g 0} \simeq c_{s}\right)$, for parameters typical of the magnetosphere $: \omega_{p} / \omega_{c}=2$, $\omega_{0} / \omega_{c}=0.96, c k_{0} / \omega_{c}=10, B / B_{0}=0.01, c_{s}=v_{g 0}=0.0073$.

In Fig. 2 we present results of numerical simulations performed for the parameters of Fig. 1 (middle panel). It shows that not only large amplitude whistlers but also smaller ones $\left(B / B_{0} \sim 0.01\right)$ may be subject to modulational instabilities; however, in the latter cases, the actual observation of such instabilities in space plasmas could be difficult if not impossible, due to the small value of $\operatorname{Im}\left(\Omega / \omega_{0}\right)$. In the left column of Fig. 2 one can see the profiles of the magnetic field envelope $B / B_{0}$ (together with its real and imaginary parts) for four moments of time, i.e. $\omega_{c} t=1.710^{8}, 1.910^{8}, 2.510^{8}$, and $310^{8}$. A modulation instability develops near $\omega_{c} t \simeq 10^{8}$, as expected from the growth rate values of Fig. 1 (middle panel). Correspondingly, the right column of Fig. 2 shows for the same time moments the profiles of the lower frequency perturbations, i.e. the normalized plasma fluid velocity $V / c_{s}$. The modulation instability manifests itself by the occurrence of nonlinear wave structures forming a set of several soliton-like peaks, which are shown in the frame moving with a velocity close to the group velocity $v_{g 0}$ where they are quasi-immobile. The distance between 

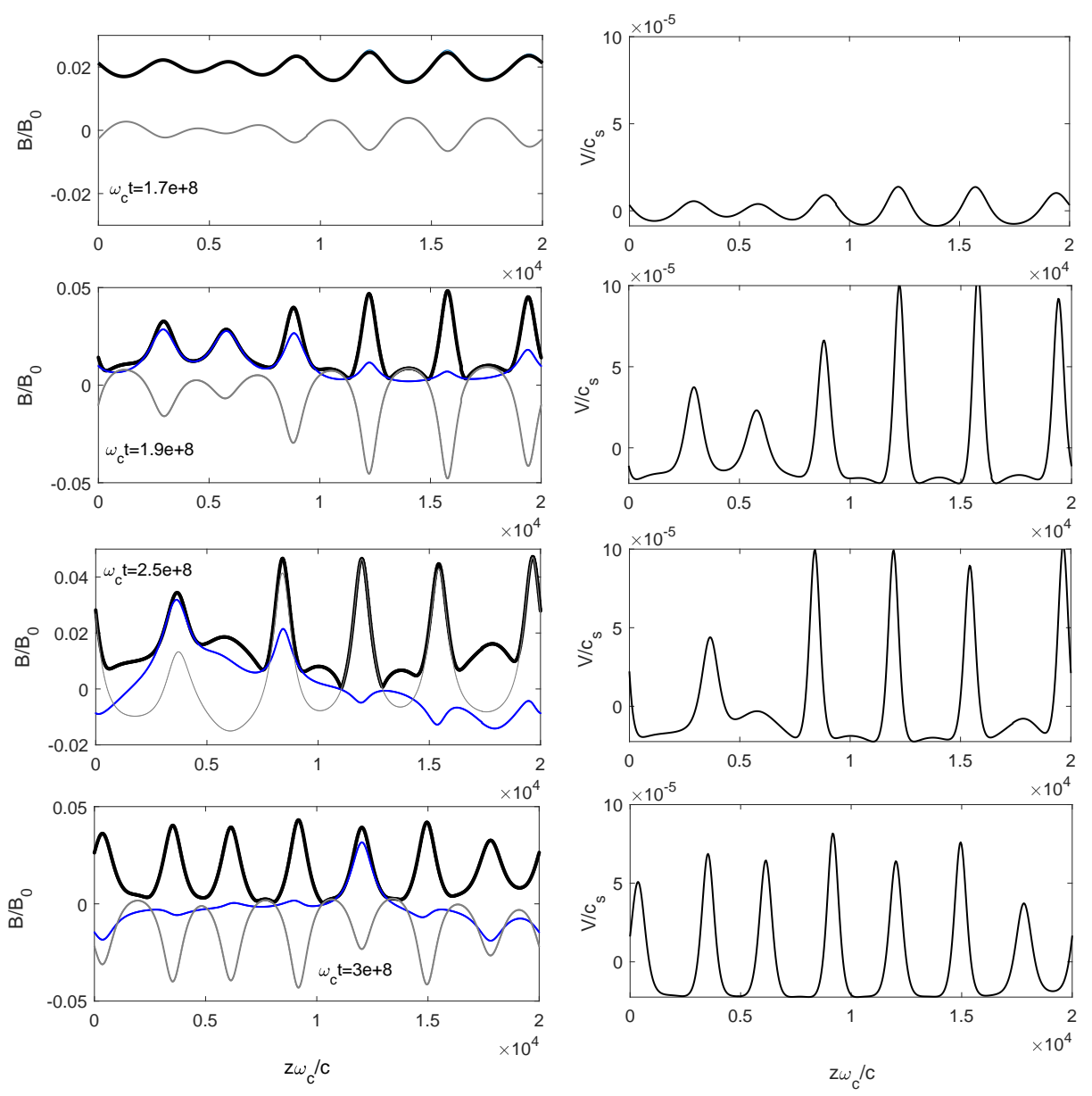

FIG. 2. (Color online) (Left column) Profiles of the magnetic field envelope $B / B_{0}$ (thick black curve), its real and imaginary parts (thin black and gray lines - blue and gray lines online respectively), for four moments of time, i.e. $\omega_{c} t=1.710^{8}, 1.910^{8}, 2.510^{8}$, and $310^{8}$. (Right column) Corresponding profiles of the lower frequency fluid velocity perturbation $V / c_{s}$, at the same times. Parameters are those of Fig. 1, middle panel.

them $\left(\Lambda \simeq 3500 c / \omega_{c}\right)$ corresponds to the wavelength of the most unstable perturbation in Fig. 1 (middle panel), i.e. to the wavenumber value $\kappa_{m} / k_{0}=0.00045$ where the growth rate is maximum; indeed one easily verifies that $2 \pi / \kappa_{m} \simeq 3700 c / \omega_{c}$. This distance $\Lambda$ is not very large in comparison with the normalized width of a soliton-like structure, which is of the order of $1000 c / \omega_{c}$. As a consequence, it is more reasonable to interpret the nonlinear structures as a periodic nonlinear wave, and not as a soliton or a train of solitons. Moreover the simulation demonstrates a more complicated picture: the growth of the modulational instability leads to the generation of a superposition (or beating) of nonlinear waves which are responsible for the large quasiperiodic oscillations of the ion sound energy density $W_{I S}$ during its saturation stage, which starts near $\omega_{c} t \simeq 1.510^{8}$, as shown in Fig. 3. Note that 


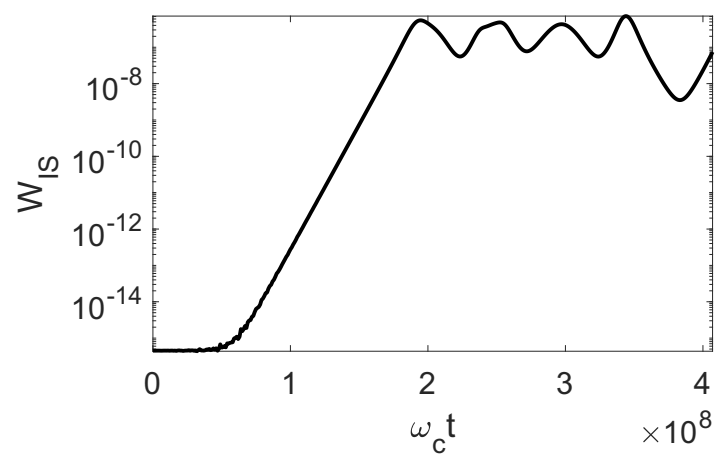

FIG. 3. Time variation of the normalized ion sound energy density $W_{I S}$ (in logarithmic scale). Parameters are those of Fig. 2.

during these oscillations the whistler wave modulation tends to disappear (see e.g. the third left panel of Fig. 2 representing $B / B_{0}$ at $\omega_{c} t=2.510^{8}$ ) as it is roughly 10 times smaller than its maximum value at $\omega_{c} t \simeq 310^{8}$. An attempt to describe theoretically such interactions between nonlinear waves was presented in Ref. 29.

\section{WHISTLER ENVELOPE SOLITON}

This Section is devoted to determine the analytical form of a whistler envelope soliton and then to study, owing to numerical simulations, its characteristics of propagation, i.e. mainly its dynamics in a inhomogeneous plasma and its collision with another soliton. Therefore let us find a stationary solution of the equations (6), (12) and (13). We suppose that the whistler envelope soliton is moving with the velocity $U$ and define the variable $\xi=z-U t$, so that for a function $f(z, t)$ we can write that $\partial f / \partial t=-U \partial f / \partial z$. Then (12) and (13) provide that $\rho=V / U$ and

$$
\rho(B)=\frac{\omega_{0}}{\left(\omega_{0}-\omega_{c}\right)} \frac{\omega_{p}^{2}}{k_{0}^{2}\left(U^{2}-c_{s}^{2}\right)}\left(1+\frac{\omega_{c}}{\left(\omega_{0}-\omega_{c}\right)} \frac{k_{0} U}{\omega_{0}}\right) \frac{|B|^{2}}{16 \pi n_{0} m_{i} c^{2}} .
$$

Moreover, searching stationary solutions of (6) in the form $B=\Phi(\xi) e^{-i \lambda t+i \mu z}$, we get that

$$
\Phi^{\prime \prime}+\frac{2}{v_{g 0}^{\prime}}\left(\lambda-\mu v_{g 0}-\mu^{2} \frac{v_{g 0}^{\prime}}{2}\right) \Phi+b \Phi^{3}=0
$$

where $\Phi^{\prime \prime}=\partial^{2} \Phi / \partial^{2} \xi$ and

$$
b=-\frac{2 \omega_{p}^{4}}{v_{g 0}^{\prime} \mathcal{D}_{0}^{\prime}\left(\omega_{0}-\omega_{c}\right)^{2}} \frac{\omega_{0}^{2}}{k_{0}^{2}\left(c_{s}^{2}-U^{2}\right)}\left(1+\frac{\omega_{c}}{\omega_{0}-\omega_{c}} \frac{k_{0} U}{\omega_{0}}\right)^{2} \frac{1}{16 \pi n_{0} m_{i} c^{2}} .
$$

One can find a family of solutions of $(31)$ in the form $\Phi(\xi)=B_{s} \sec \left(\xi / l_{s}\right)$ with

$$
\mu=\frac{U-v_{g 0}}{v_{g 0}^{\prime}}, \quad \lambda=-\frac{v_{g 0}^{\prime}}{2 l_{s}^{2}}+\mu v_{g 0}+\mu^{2} \frac{v_{g 0}^{\prime}}{2},
$$




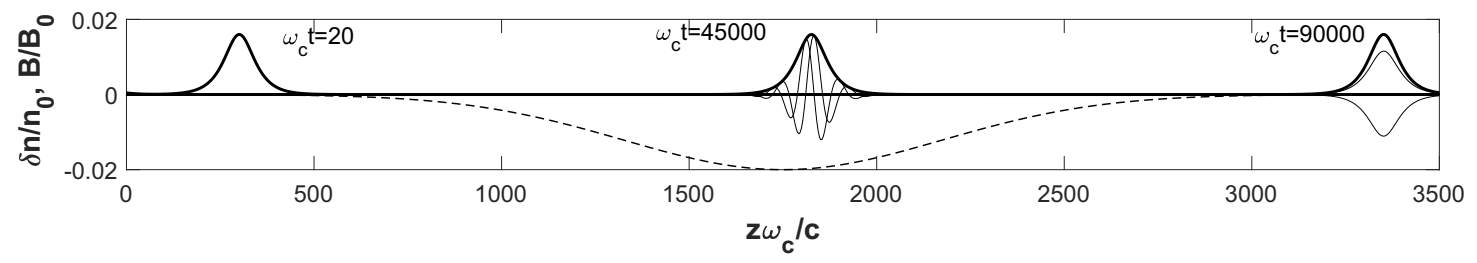

FIG. 4. Whistler envelope soliton (in thick lines) at three time moments $\omega_{c} t=20,45000$ and 90000 during its motion through a density depletion reaching $\delta n / n_{0} \simeq-0.02$ (dashed lines); phase oscillations inside the envelope are presented in thin lines. Main parameters are : $\omega_{p} / \omega_{c}=17$, $\omega_{0} / \omega_{c}=0.41, c k_{0} / \omega_{c}=14.3, B / B_{0}=0.015, c_{s}=0.00014, v_{g 0}=0.0034$.

and

$$
l_{s}^{2}=\frac{16 \pi n_{0} m_{i} c^{2}}{B_{s}^{2}} \frac{\left(\omega_{0}-\omega_{c}\right)^{2}}{\omega_{p}^{4}} \frac{2 k_{0} v_{g 0}^{\prime}}{v_{g 0}} \frac{c^{2} k_{0}^{2}}{\omega_{0}^{2}}\left(c_{s}^{2}-U^{2}\right)\left(1+\frac{\omega_{c}}{\omega_{0}-\omega_{c}} \frac{k_{0} U}{\omega_{0}}\right)^{-2} .
$$

Then the lower frequency perturbations are given by $\rho=\rho_{0} \sec ^{2}\left(\xi / l_{s}\right)$ and $V=V_{0} \sec ^{2}\left(\xi / l_{s}\right)=$ $U \rho_{0} \sec ^{2}\left(\xi / l_{s}\right)$, where $\rho_{0}=\rho\left(B_{s}\right)(30)$.

Equation (34) shows that the whistler soliton exists only if $v_{g 0}^{\prime}\left(c_{s}^{2}-U^{2}\right)>0$. So the condition $v_{g 0}^{\prime}<0$ (resp. $v_{g 0}^{\prime}>0$ ) has to be fulfilled for a supersonic whistler soliton with $U>c_{s}$ (resp. a subsonic soliton with $U<c_{s}$ ). One can see that the width $l_{s}$ of the soliton is inversely proportional to its amplitude $B_{s}$. For a supersonic soliton, $\rho$ and $V$ propagate in the form of density and velocity humps if

$$
\frac{\omega_{0}}{c k_{0}} \frac{\left(\omega_{c}-\omega_{0}\right)}{\omega_{c}}<\frac{U}{c}
$$

and in the form of cavities for the opposite case. When the soliton velocity is equal to the group velocity, i.e. $U=v_{g 0}$, one can show using (2) and (7) that the term $1+\omega_{c} k_{0} U / \omega_{0} /\left(\omega_{0}-\omega_{c}\right)$ is always negative, so that the supersonic solitary whistler wave presents in this case always density and velocity humps and the subsonic one always density and velocity cavities. Note that the difference between $U$ and the group velocity $v_{g 0}$ is formal, as it becomes clear if one notes that a soliton moving with velocity $U \neq v_{g 0}$ gets a correction $\mu$ to its wavenumber (33); thus the soliton always moves with the group velocity corresponding to its full wavenumber $\mu+k_{0}$. But it is suitable to distinguish $U$ and $v_{g 0}$ when we consider the collision of two solitons (see below).

The questions arise whether a inhomogeneous plasma can support the propagation of stable whistler solitons and whether they can be accelerated or decelerated in such a medium, as a linear whistler wave does, and if they can keep their identities when passing through density gradients or randomly fluctuating plasma irregularities. So let us study the propagation of a whistler soliton in a depleted plasma with a density well of depth reaching $2 \%$ of the ambient plasma density and a scale significantly larger than the soliton width. Numerical simulations show that the solitary whistler propagating in this structure keeps its stability. 

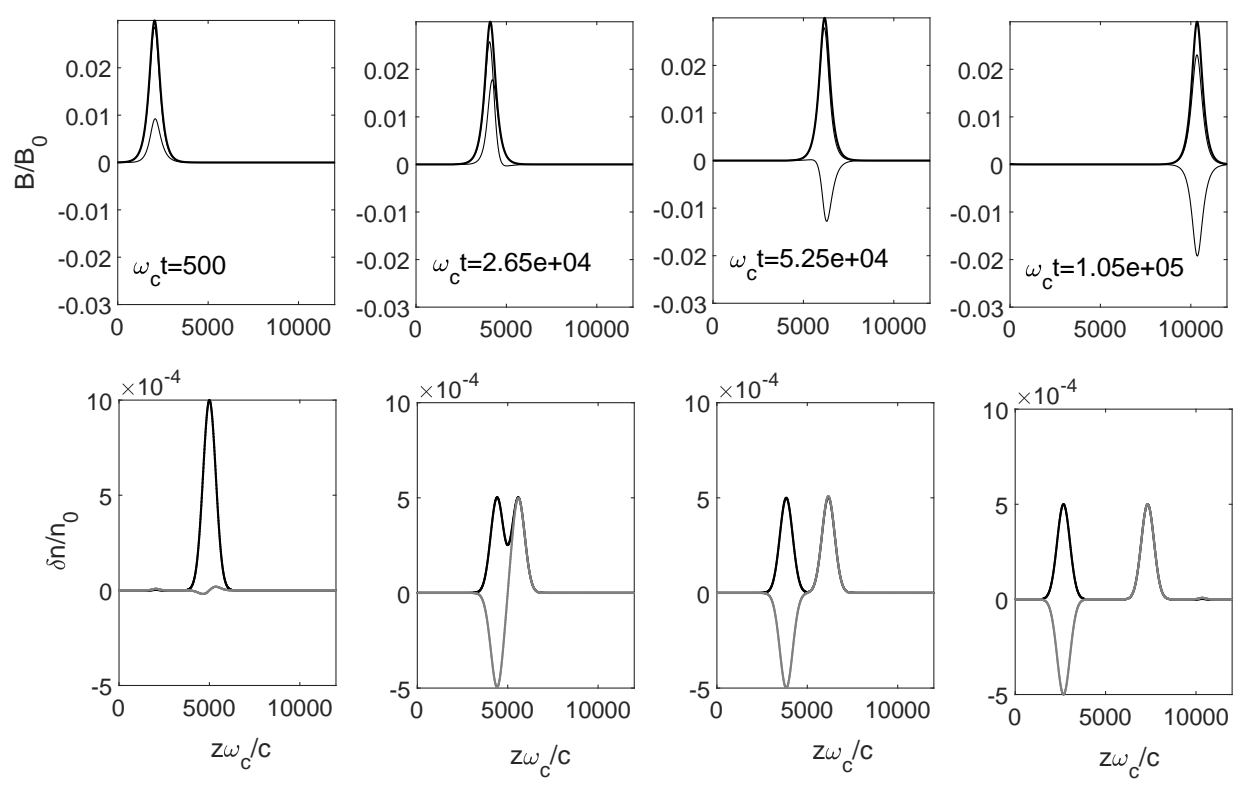

FIG. 5. (Upper row) Whistler envelope soliton (thick black lines) at four time moments $\omega_{c} t=500$, $2.6510^{4}, 5.2510^{4}, 1.0510^{5}$ during its motion through a density hump with maximum $\delta n / n_{0} \simeq 0.001$ (bottom row, thick black lines); the phase oscillations inside the envelope are presented in thin black lines. (Bottom row) : Corresponding profiles of the external density (black lines) and velocity (gray lines) perturbations. In all places where black and gray lines do not appear separately, they are actually superposed. Main parameters are : $\omega_{p} / \omega_{c}=3, \omega_{0} / \omega_{c}=0.73, c k_{0} / \omega_{c}=5.0, B / B_{0}=0.03$, $c_{s}=0.02, v_{g 0}=0.079$.

Figure 4 presents the profiles of the whistler envelope soliton $B / B_{0}$ at three different times and positions when propagating through a small density depletion. The envelope is not deformed during its travel but its phase exhibits oscillations with varying wavelengths; those decrease more and more as the soliton approaches the bottom of the density well. The soliton moves with a quasi-constant velocity, it decelerates (resp. accelerates) when it crosses the negative (resp. positive) density gradient, in agreement with the behavior expected for a linear wave. When it reaches the simulation box at $\omega_{c} t \sim 10^{5}$, its velocity has eventually increased by less than $1 \%$. These variations are tiny due to the small corresponding variations of the whistler frequency.

The dynamics of an envelope soliton crossing a density hump of comparable width is shown in Fig. 5 at four time moments, together with the corresponding evolutions of the density and the velocity external perturbations (the soliton's lower frequency perturbations are too small to be visible). When the soliton passes through the hump (see the second column), the external density hump splits in two parts of identical size but of half amplitude that are propagating further in opposite directions; moreover the corresponding velocity 

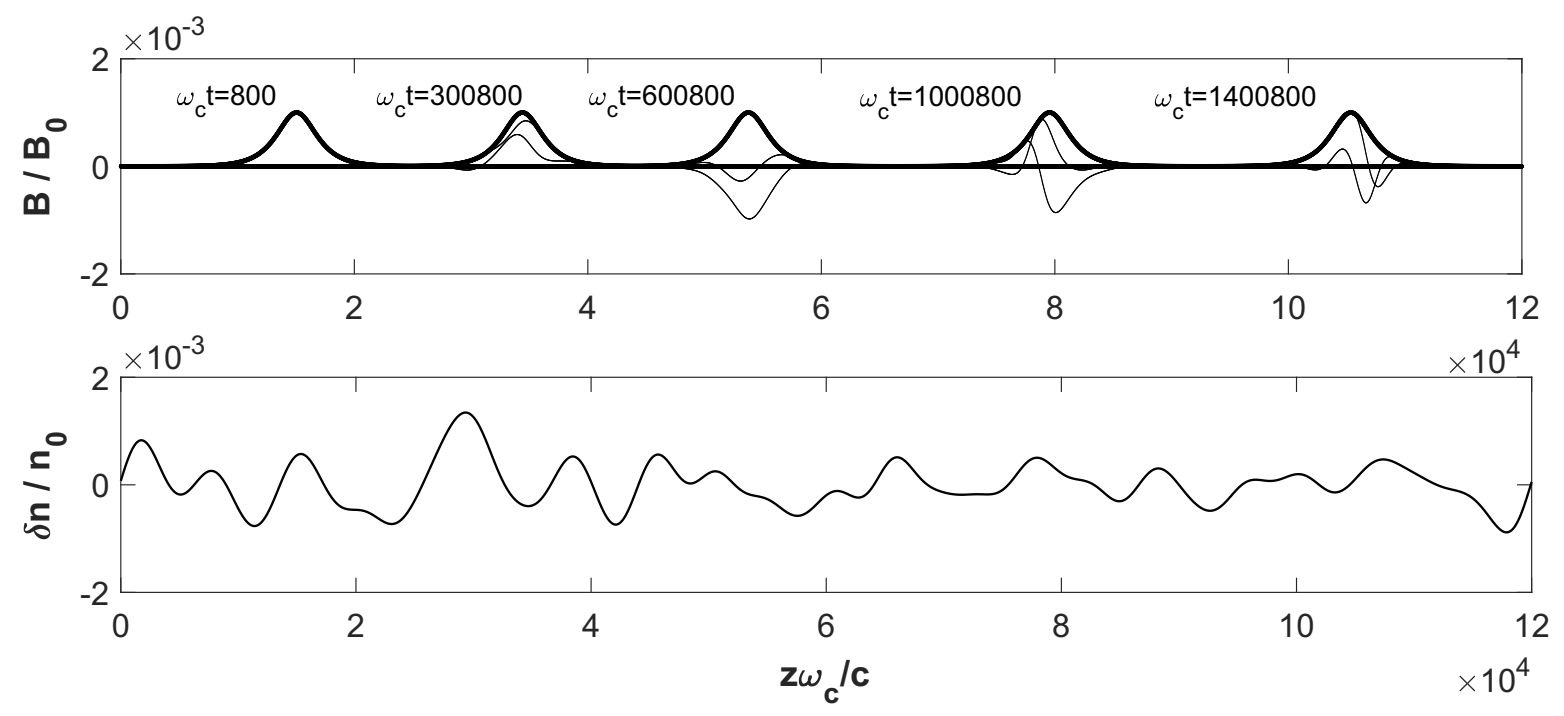

FIG. 6. (Upper panel) : Whistler envelope soliton $B / B_{0}$ (thick lines) at five time moments $\omega_{c} t=$ $800,300800,600800,1000800$, and 1400800 during its motion through an inhomogeneous plasma with random density fluctuations; phase oscillations inside the envelope are presented in thin lines. (Lower panel) : Profile of the external density fluctuations $\delta n / n_{0}$. Main parameters are : $\omega_{p} / \omega_{c}=10, \omega_{0} / \omega_{c}=0.28, c k_{0} / \omega_{c}=6.3, B / B_{0}=0.001, c_{s}=0.00015, v_{g 0}=0.065$.

perturbation is amplified and appears as a hump accompanied by a depletion of similar scale and amplitude. Meanwhile the soliton envelope keeps its stability, even if its phase strongly varies.

Moreover, the propagation of a whistler envelope soliton in a plasma with random density fluctuations of comparable amplitudes and wavelengths has been studied, showing that the soliton is very weakly affected by these irregularities. Fig. 6 shows the soliton profile at five different time moments together with the density fluctuations' profile $\delta n / n_{0}$. Note that such kind of plasma with random density inhomogeneities is typical of the solar wind. As discussed above, only the phase of the soliton is strongly varying when it crosses successive irregularities with negative or positive gradients, its envelope remaining very stable. The soliton is not accelerated nor decelerated during its travel through the whole box, i.e. during a time exceeding $1.510^{6} \omega_{c}^{-1}$.

Finally, simulations show for the first time that two whistler solitons moving with different but close velocities and colliding one with the other are not destroyed during this process but propagate away with no changes after the collision is fulfilled, independently of their amplitudes and velocities. Figure 7 presents the magnetic field amplitudes $B / B_{0}$ of two envelope solitons moving along the simulation box with different velocities (both very close to the group velocity), at six different times during the collision. When the two solitons begin to overlap, their envelopes as well as the oscillations of their phases are strongly modified, 

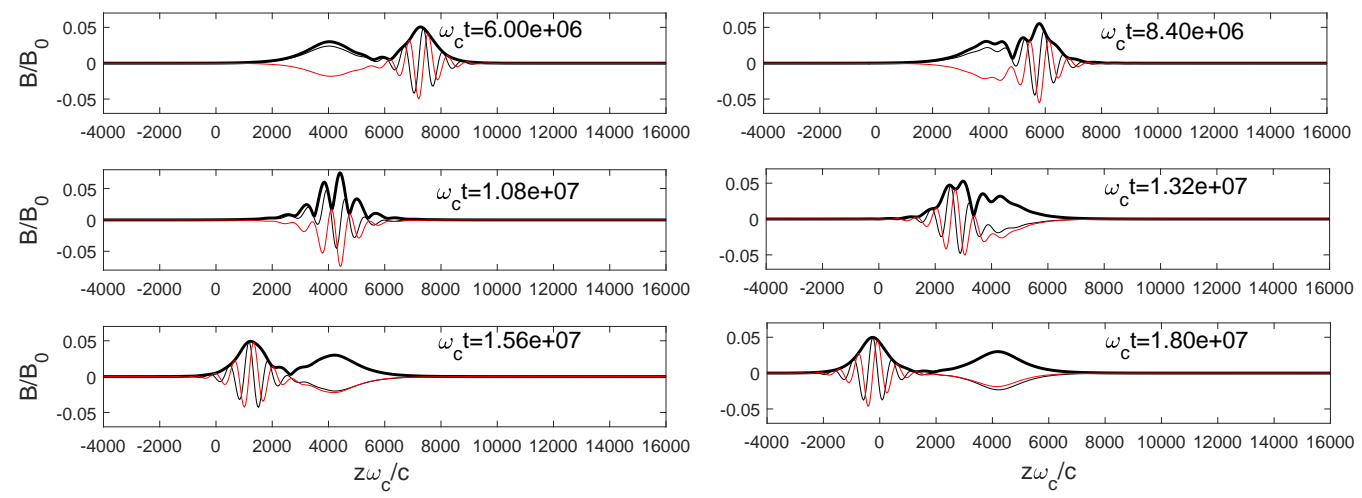

FIG. 7. (Color online) Time evolution of the collision between two whistler envelope solitons. Six moments of time are shown, as indicated on each panel : $\omega_{c} t=610^{6}, 8.410^{6}, 1.0810^{7}, 1.3210^{7}$, $1.5710^{7}$, and $1.810^{7}$. The first soliton, the fastest one, has the amplitude $B / B_{0}=0.03$ (upper left panel); the second soliton, the slowest one, has the amplitude $B / B_{0}=0.05$. The envelopes are shown in thick black lines, whereas the real and imaginary parts are presented in thin and dotted (red online) lines. Main parameters are : $\omega_{p} / \omega_{c}=3, \omega_{0} / \omega_{c}=0.49, c k_{0} / \omega_{c}=3, c_{s}=0.001$, $v_{g 0}=0.17$.

interfering one with the other in a complex way. Note however that the wavelength of the real and imaginary parts of the fields are not changed; the phase oscillations with the smallest wavelength (belonging to the slowest soliton) modulate the phase oscillations with the longest wavelength (the fastest envelope). At the time $\omega_{c} t \simeq 1.0810^{7}$ when the solitons coalesce, they exhibit a single structure with several peaks of wavelengths close to those of the slowest soliton. After the collision, when the fastest soliton (i.e. that with the smallest amplitude) has overtaken the slowest one (with the highest amplitude), both recover their initial shapes and continue their motion without noticeable variations of their amplitudes, forms and phases. The density and the velocity perturbations (not shown here) propagate in the form of $\sec ^{2}\left(\xi / l_{s}\right)$ functions as determined analytically, following the whistler soliton with the same speeds. However, similarly to the case of Langmuir solitons' collisions, emission of linear ion sound waves is detected after the collision is finished.

In a second example (see Fig. 8), the fastest soliton in its turn has the largest amplitude, but the conclusions are the same as mentioned previously. Moreover we present in Fig. 9 the time variations of the square maximum amplitude $\left(B_{\max } / B_{0}\right)^{2}$ of the overlapped structure formed by the two solitons and of its position $\xi=z_{\max }-v_{g 0} t$ in the frame moving with the group velocity, where $z=z_{\max }$ for $B=B_{\max }$. This shows that the structure moves with a constant velocity and that its maximum amplitude oscillates weakly with time. 

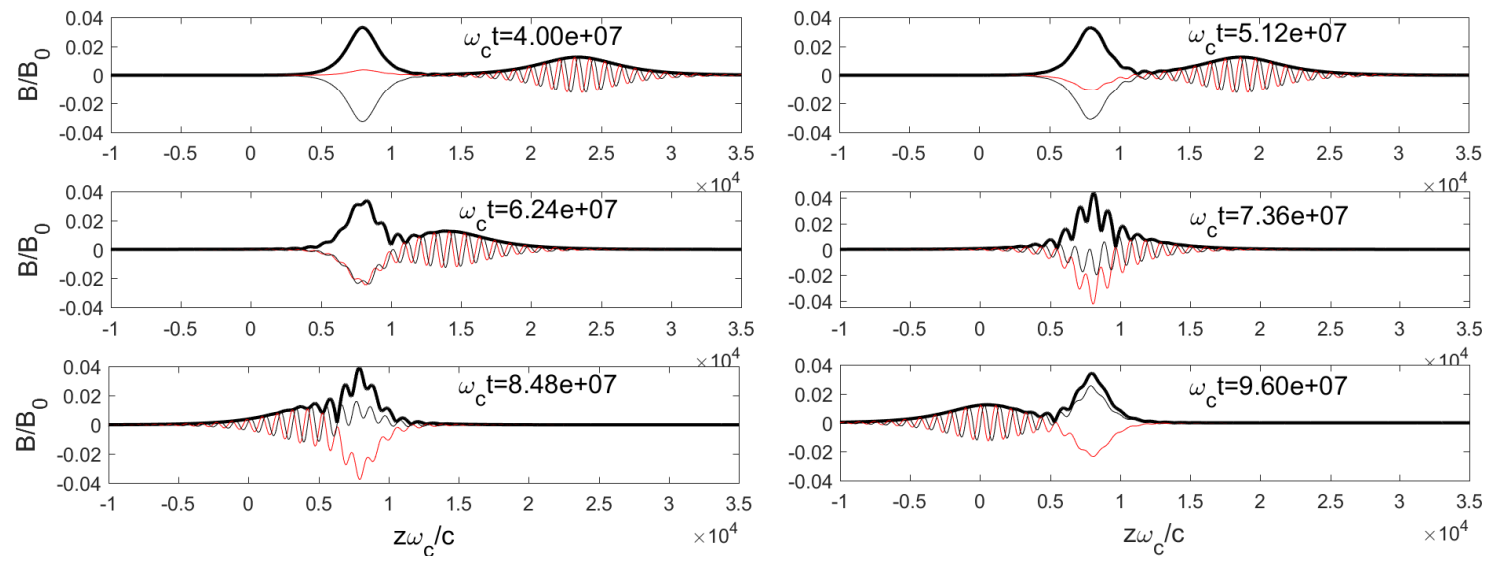

FIG. 8. (Color online) Time evolution of the collision between two whistler envelope solitons. Six moments of time are shown, as indicated on each panel : $\omega_{c} t=410^{7}, 5.1210^{7}, 6.2410^{7}, 7.3610^{7}$, $8.4810^{7}$, and $9.610^{7}$. The first soliton, the fastest one, has the amplitude $B / B_{0}=0.03$ (upper left panel); the second soliton, the slowest one, has the amplitude $B / B_{0}=0.01$. The envelopes are shown in thick black lines, whereas the real and imaginary parts are presented in thin and dotted (red online) lines. Main parameters are : $\omega_{p} / \omega_{c}=3,, \omega_{0} / \omega_{c}=0.63, c k_{0} / \omega_{c}=4, c_{s}=0.001, v_{g 0}=$ 0.117 .

\section{SONIC WHISTLER SOLITON}

When the whistler group velocity $v_{g 0}$ is very close to the ion sound velocity $c_{s}$, the width $l_{s}$ of the soliton (34) becomes infinitely small and its amplitude $E_{s}$ infinitely large. In order to suppress this singularity, we have to take into account small additional corrections in the low frequency equations (hydrodynamic nonlinearities and weak non quasineutrality). Let us consider that $v_{g 0} \simeq c_{s}$ and combine the low frequency equations (10)-(11) as follows

$$
\frac{\partial^{2} \rho}{\partial t^{2}}-\frac{T_{i}}{m_{i}} \frac{\partial^{2}}{\partial z^{2}}\left(\rho+\frac{e \varphi}{T_{i}}\right)=\frac{\partial}{\partial z}\left(V \frac{\partial V}{\partial z}-\frac{\partial}{\partial t}(\rho V)\right)
$$

where we used the same notations $V$ and $\rho=\delta n_{i} / n_{0}=\left(n_{i}-n_{0}\right) / n_{0}$ as in the previous Section; $\varphi$ is the low frequency potential. The density $n_{h}$ of the electrons, which are a hot population compared to the ions, is

$$
n_{h}=\int_{-\infty}^{\infty}\left(f^{(0)}+f^{(1)}\right) d v_{z}=n_{h}^{(0)}+n_{h}^{(1)},
$$

where $n_{h}^{(0)}$ and $n_{h}^{(1)}$ are the zero and first order electron densities, corresponding to the parallel velocity distributions $f^{(0)}$ and $f^{(1)} ; n_{h}^{(0)}$ is obtained by writing that the resulting force acting on the hot electrons (Coulomb, thermal and stationary ponderomotive forces) is vanishing, which leads to

$$
n_{h}^{(0)} \simeq n_{h 0} \exp \left(\frac{e \varphi}{T_{h}}-\frac{\omega_{p}^{2}}{\omega_{0}\left(\omega_{0}-\omega_{c}\right)} \frac{|E|^{2}}{16 \pi n_{0} T_{h}}\right),
$$



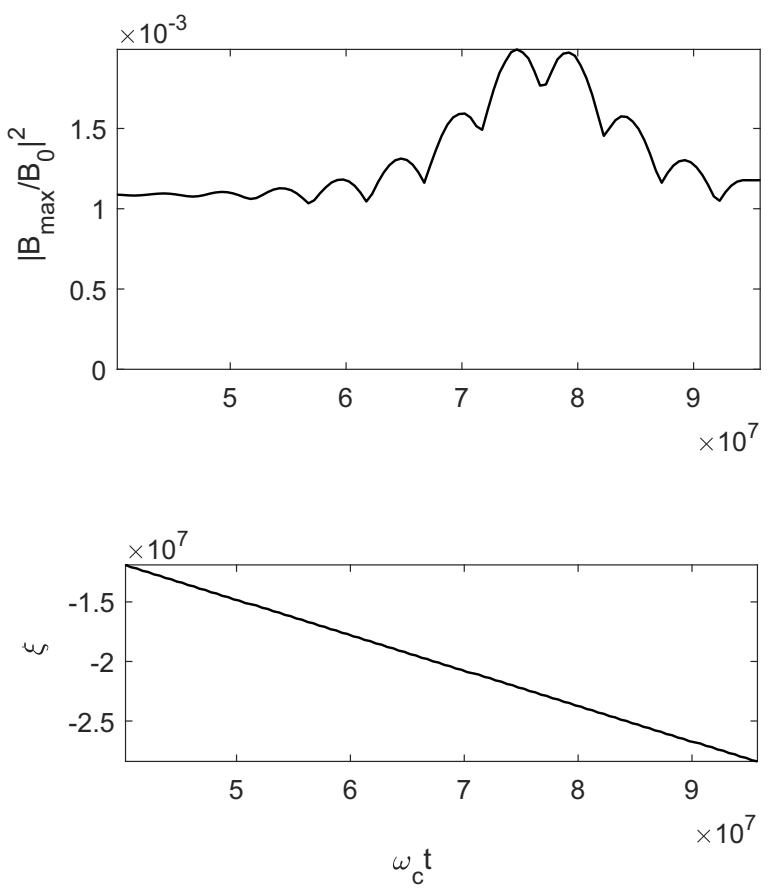

FIG. 9. (Upper panel) : Time variation, during the collision, of the square maximum amplitude $\left(B_{\max } / B_{0}\right)^{2}$ of the structure formed by the two overlapped solitons in Fig. 8. (Lower panel) : Corresponding time variation of the position $\xi=z_{\max }-v_{g 0} t$ in the frame moving with the group velocity, where $z=z_{\max }$ for $B=B_{\max }$. Parameters are those of Fig. 8 .

where $T_{h}$ is the temperature of the electron population and $n_{h 0}$ is the electron density at equilibrium. Considering the higher order terms provided by the thermal force and the non stationary part of the ponderomotive force, we get similarly the derivative of the first order electron density

$$
\frac{\partial n_{h}^{(1)}}{\partial z} \simeq n_{h 0} \frac{\omega_{p}^{2} \omega_{c}}{\omega_{0}\left(\omega_{0}-\omega_{c}\right)^{2}} \frac{k_{0}}{\omega_{0}} \frac{\partial}{\partial t} \frac{|E|^{2}}{16 \pi n_{0} T_{h}} .
$$

The Poisson equation can be written as

$$
\frac{\partial^{2} \varphi}{\partial z^{2}}=-4 \pi e\left(n_{i}-n_{h}\right)=-4 \pi e\left(\rho n_{0}-\left(n_{h}^{(0)}+n_{h}^{(1)}-n_{0}\right)\right)
$$

which leads to

$$
\left(1-\lambda_{h}^{2} \frac{\partial^{2}}{\partial z^{2}}\right) \frac{e \varphi}{T_{h}}=\rho+\frac{\omega_{p}^{2}}{\omega_{0}\left(\omega_{0}-\omega_{c}\right)} \frac{|E|^{2}}{16 \pi n_{0} T_{h}}+\frac{1}{2}\left(\frac{e \varphi}{T_{h}}-\frac{\omega_{p}^{2}}{\omega_{0}\left(\omega_{0}-\omega_{c}\right)} \frac{|E|^{2}}{16 \pi n_{0} T_{h}}\right)^{2}-\frac{n_{h}^{(1)}}{n_{0}},
$$

where $\lambda_{h}^{2}=T_{h} / 4 \pi n_{0} e^{2}$ is the electron Debye length. Excluding $\varphi$ in the right hand side of (41) we get

$$
\frac{\partial}{\partial z} \frac{e \varphi}{T_{h}} \simeq\left(1+\lambda_{h}^{2} \frac{\partial^{2}}{\partial z^{2}}\right) \frac{\partial \rho}{\partial z}+\frac{\omega_{p}^{2}}{\omega_{0}\left(\omega_{0}-\omega_{c}\right)}\left(\frac{\partial}{\partial z}-\frac{\omega_{c}}{\omega_{0}-\omega_{c}} \frac{k_{0}}{\omega_{0}} \frac{\partial}{\partial t}\right) \frac{|E|^{2}}{16 \pi n_{0} T_{h}}+\frac{1}{2} \frac{\partial \rho^{2}}{\partial z} .
$$


Then, using (36) we obtain that

$$
\begin{gathered}
\frac{\partial^{2} \rho}{\partial t^{2}}-c_{s}^{2} \frac{\partial^{2} \rho}{\partial z^{2}}-c_{s}^{2} \frac{\partial^{2}}{\partial z^{2}}\left(\lambda_{0}^{2} \frac{\partial^{2} \rho}{\partial z^{2}}+\frac{T_{h}}{T_{h}+T_{i}} \frac{\rho^{2}}{2}\right) \\
-\frac{\omega_{p}^{2}}{\omega_{0}\left(\omega_{0}-\omega_{c}\right)} \frac{\partial}{\partial z}\left(\frac{\partial}{\partial z}-\frac{\omega_{c}}{\left(\omega_{0}-\omega_{c}\right)} \frac{k_{0}}{\omega_{0}} \frac{\partial}{\partial t}\right) \frac{|E|^{2}}{16 \pi n_{0} m_{i}}=\frac{\partial}{\partial z}\left(V \frac{\partial V}{\partial z}-\frac{\partial}{\partial t}(\rho V)\right),
\end{gathered}
$$

where $\lambda_{0}^{2}=T_{h} \lambda_{h}^{2} /\left(T_{h}+T_{i}\right)$. For waves propagating along one direction with the velocity $U$ we can write that $\rho \simeq \rho(\tau, \xi)$, where $\xi=z-U t$, and suppose the slow dependence of $\rho$ on $\tau$ (i.e. $\partial / \partial t \simeq-U \partial / \partial z$ ). In the first approximation we get that $V \simeq \rho U$ and, as we can write that

$$
\frac{\partial}{\partial z}\left(V \frac{\partial V}{\partial z}-\frac{\partial}{\partial t}(\rho V)\right) \simeq \frac{3}{2} U^{2} \frac{\partial^{2} \rho^{2}}{\partial z^{2}}, \quad \frac{\partial^{2} \rho}{\partial t^{2}} \simeq-2 U \frac{\partial}{\partial z} \frac{\partial \rho}{\partial \tau}+U^{2} \frac{\partial^{2} \rho}{\partial z^{2}}
$$

we can derive that

$$
\begin{gathered}
\frac{\partial \rho}{\partial \tau}-\frac{\left(U^{2}-c_{s}^{2}\right)}{2 U} \frac{\partial \rho}{\partial z}+\frac{c_{s}^{2}}{2 U} \frac{\partial}{\partial z}\left(\lambda_{0}^{2} \frac{\partial^{2} \rho}{\partial z^{2}}+\frac{\lambda_{0}^{2}}{\lambda_{h}^{2}} \frac{\rho^{2}}{2}\right) \\
+\frac{\omega_{p}^{2}}{2 U \omega_{0}\left(\omega_{0}-\omega_{c}\right)}\left(\frac{\partial}{\partial z}-\frac{\omega_{c}}{\left(\omega_{0}-\omega_{c}\right)} \frac{k_{0}}{\omega_{0}} \frac{\partial}{\partial t}\right) \frac{|E|^{2}}{16 \pi n_{0} m_{i}}+\frac{3 U}{4} \frac{\partial \rho^{2}}{\partial z}=0 .
\end{gathered}
$$

With the required accuracy we can write that $U \simeq c_{s}$ and $\partial / \partial t \simeq-c_{s} \partial / \partial z$, so that we obtain finally

$$
\frac{\partial \rho}{\partial \tau}+\frac{c_{s}}{2} \lambda_{0}^{2} \frac{\partial^{3} \rho}{\partial z^{3}}+\frac{c_{s}}{2} \frac{\partial}{\partial z}\left(\frac{c_{s}^{2}-U^{2}}{c_{s}^{2}} \rho+\left(\frac{\lambda_{0}^{2}}{\lambda_{h}^{2}}+3\right) \frac{\rho^{2}}{2}\right)=-\frac{c_{s} \chi}{2} \frac{\partial}{\partial z} \frac{|E|^{2}}{16 \pi n_{0} m_{i} c_{s}^{2}},
$$

which is a $\mathrm{KdV}$ equation with a right hand side term describing the ponderomotive effects.

The high frequency equation (6) coupled with (46) can be written in the form

$$
i \frac{\partial B}{\partial t}+i v_{g 0} \frac{\partial B}{\partial z}+\frac{v_{g 0}^{\prime}}{2} \frac{\partial^{2} B}{\partial z^{2}}=-\frac{\omega_{0} \omega_{p}^{2}}{\mathcal{D}_{0}^{\prime}\left(\omega_{0}-\omega_{c}\right)}\left(1+\frac{\omega_{c}}{\omega_{0}-\omega_{c}} \frac{k_{0} c_{s}}{\omega_{0}}\right) \rho B=\frac{\alpha \chi}{2} \rho B
$$

where we note

$$
\chi=\frac{\omega_{p}^{2}}{\omega_{0}\left(\omega_{0}-\omega_{c}\right)}\left(1+\frac{\omega_{c}}{\left(\omega_{0}-\omega_{c}\right)} \frac{k_{0} c_{s}}{\omega_{0}}\right), \quad \alpha=-\frac{2 \omega_{0}^{2}}{\mathcal{D}_{0}^{\prime}} .
$$

The system formed by the equations (46)-(47) has an Hamiltonian $H_{s}$ which is the sum of three Hamiltonians, i.e. $H_{w}, H_{\rho}$ and $H_{\text {int }}$. Indeed, multiplying (47) by $-\partial B^{*} / \partial t$, adding the complex conjugate and integrating on space, we obtain the first part $H_{w}$ of the total Hamiltonian $H_{s}$

$$
H_{w}=\int \frac{d z}{L}\left(\frac{i v_{g 0}}{2}\left(B \frac{\partial B^{*}}{\partial z}-B^{*} \frac{\partial B}{\partial z}\right)+\frac{v_{g 0}^{\prime}}{2}\left|\frac{\partial B}{\partial z}\right|^{2}\right)
$$


which is the whistler wave energy. The KdV equation (46) provides the following Hamiltonian, which is related to the energy of the low frequency dynamics

$$
H_{\rho}=\alpha \gamma \int \frac{d z}{L}\left(\left(\frac{c_{s}^{2}-U^{2}}{c_{s}^{2}}\right) \frac{\rho^{2}}{2}-\frac{\lambda_{0}^{2}}{2}\left(\frac{\partial \rho}{\partial z}\right)^{2}+\left(\frac{\lambda_{0}^{2}}{\lambda_{h}^{2}}+3\right) \frac{\rho^{3}}{6}\right),
$$

where $\gamma=8 \pi\left(c^{2} k_{0}^{2} / \omega_{0}^{2}\right) n_{0} m_{i} c_{s}^{2}$, whereas the interaction of the plasma fluid and the whistler field is governed by the third Hamiltonian

$$
H_{\text {int }}=\frac{\alpha \chi}{2} \int \frac{d z}{L} \rho|B|^{2}
$$

The total Hamiltonian $H_{s}=H_{w}+H_{i n t}+H_{\rho}$ of the system is given by

$$
\begin{gathered}
H_{s}=\int \frac{d z}{L}\left(\frac{i v_{g 0}}{2}\left(B \frac{\partial B^{*}}{\partial z}-B^{*} \frac{\partial B}{\partial z}\right)+\frac{v_{g 0}^{\prime}}{2}\left|\frac{\partial B}{\partial z}\right|^{2}\right) \\
+\int \frac{d z}{L}\left(\frac{\alpha \chi}{2} \rho|B|^{2}+\alpha \gamma\left(\left(\frac{c_{s}^{2}-U^{2}}{c_{s}^{2}}\right) \frac{\rho^{2}}{2}-\frac{\lambda_{0}^{2}}{2}\left(\frac{\partial \rho}{\partial z}\right)^{2}+\left(\frac{T_{h}}{T_{h}+T_{i}}+3\right) \frac{\rho^{3}}{6}\right)\right),
\end{gathered}
$$

where the canonical variables are the couples $\left(B, B^{*}\right)$ and $(\rho, \psi)$, with $\psi=2 \alpha \gamma \rho / c_{s}$, which satisfy the Hamilton equations

$$
\begin{gathered}
\frac{\partial B}{\partial t}=-i \frac{\delta H_{s}}{\delta B^{*}}, \quad \frac{\partial B^{*}}{\partial t}=i \frac{\delta H_{s}}{\delta B} \\
\frac{\partial \rho}{\partial t}=-\frac{\partial}{\partial z}\left(\frac{\delta H_{s}}{\delta \psi}\right), \quad \frac{\partial \psi}{\partial t}=-\frac{\partial}{\partial z}\left(\frac{\delta H_{s}}{\delta \rho}\right) .
\end{gathered}
$$

In order to determine the form of the soliton which can propagate in the system, let us define the variables $\tau=t$ and $\xi=z-v_{g 0} t$ and write the equations (46)-(47) in the frame moving with the group velocity as

$$
\begin{gathered}
\frac{\partial \rho}{\partial \tau}+\left(c_{s}-v_{g 0}\right) \frac{\partial \rho}{\partial \xi}+\frac{c_{s}}{2} \frac{\partial}{\partial \xi}\left(\lambda_{0}^{2} \frac{\partial^{2} \rho}{\partial \xi^{2}}+\frac{1}{2}\left(\frac{\lambda_{0}^{2}}{\lambda_{h}^{2}}+3\right) \rho^{2}\right)+\frac{c_{s} \chi}{2} \frac{\partial}{\partial \xi} \frac{|E|^{2}}{16 \pi n_{0} m_{i} c_{s}^{2}}=0 \\
i \frac{\partial E}{\partial \tau}+\frac{v_{g 0}^{\prime}}{2} \frac{\partial^{2} E}{\partial \xi^{2}}=\frac{\alpha \chi}{2} \rho E .
\end{gathered}
$$

Let us search in this frame stationary solutions in the form $\rho=\rho(\xi)$ and $E=E_{s}(\xi) e^{-i \varpi t} e^{i \mu^{\prime} z}$. We get

$$
\frac{2\left(c_{s}-v_{g 0}\right)}{c_{s}} \rho+\lambda_{0}^{2} \frac{\partial^{2} \rho}{\partial \xi^{2}}+\frac{1}{2}\left(\frac{\lambda_{0}^{2}}{\lambda_{h}^{2}}+3\right) \rho^{2}+\frac{\chi\left|E_{s}\right|^{2}}{16 \pi n_{0} m_{i} c_{s}^{2}}=0,
$$

and

$$
\frac{v_{g 0}^{\prime}}{2} \frac{\partial^{2} E_{s}}{\partial \xi^{2}}+\varpi E_{s}-\frac{\alpha \chi}{2} \rho E_{s}=0
$$

Note that the expected mutual compensation of dispersive and nonlinear effects is realized in the higher frequency equation (58) but unlikely in the lower frequency equation (57), 
due to the smallness of the dispersive term $\lambda_{0}^{2} \partial^{2} \rho / \partial \xi^{2}$, which is typically 100 times smaller than $\left(v_{g 0}^{\prime} / 2\right) \partial^{2} E / \partial \xi^{2}$. In this case the nonlinear term proportional to $\rho^{2}$, which could lead to steepening effects and wave breaking, is compensated by the ponderomotive effects proportional to $\left|E_{s}\right|^{2}$. The equations (57)-(58) have solutions in the form $\rho(\xi)=\rho_{0} \sec ^{2}\left(\xi / l_{s}\right)$ and $E_{s}(\xi)= \pm E_{s 0} \sec \left(\xi / l_{s}\right) \tanh \left(\xi / l_{s}\right)$, with $\varpi=-v_{g 0}^{\prime} / 2 l_{s}^{2}$ and

$$
\rho_{0}=-\frac{6 v_{g 0}^{\prime}}{\alpha \chi l_{s}^{2}} \simeq-\frac{\left(c_{s}-v_{g 0}\right)}{c_{s}} \frac{4}{\lambda_{0}^{2} / \lambda_{h}^{2}+3-2 \alpha \chi \lambda_{0}^{2} / 3 v_{g 0}^{\prime}},
$$

where the term $2\left|\alpha \chi / v_{g 0}^{\prime}\right| \lambda_{0}^{2} / 3 \ll 1$ in the denominator can be neglected compared to the other ones. With the same accuracy we get also

$$
\begin{aligned}
\frac{\left|E_{s}\right|^{2}}{16 \pi n_{0} m_{i} c_{s}^{2}} & \simeq \frac{8\left(c_{s}-v_{g 0}\right)^{2}}{\chi c_{s}^{2}\left(\lambda_{0}^{2} / \lambda_{h}^{2}+3\right)}, \\
\frac{c^{2}}{\omega_{c}^{2} l_{s}^{2}} & \simeq \frac{\left(c_{s}-v_{g 0}\right)}{c_{s}} \frac{c^{2}}{\omega_{c}^{2}} \frac{2 \alpha \chi}{3 v_{g 0}^{\prime}\left(\lambda_{0}^{2} / \lambda_{h}^{2}+3\right)} .
\end{aligned}
$$

Note that $\alpha$ is positive whatever the parameters are and that $\chi>0$ when $v_{g 0} \simeq c_{s}$. One can show that, similarly to $\rho_{0},\left|E_{s}\right|$ and $1 / l_{s}^{2}$ are proportional to $c_{s}-v_{g 0}$ : when $v_{g 0} \rightarrow c_{s}$ the soliton's amplitude decreases and its width increases. If $v_{g 0}^{\prime}>0$, the soliton exists whatever the parameters are, it is slightly subsonic $\left(v_{g 0} \lesssim c_{s}\right)$ and propagates with a density dip $\rho_{0}<0$. On the other hand, if $v_{g 0}^{\prime}<0$, the soliton only exists if the following condition is fulfilled

$$
\frac{T_{h}}{T_{h}+T_{i}}+3>\frac{2 \alpha \chi \lambda_{0}^{2}}{\left|v_{g 0}^{\prime}\right|}
$$

In this case, it is slightly supersonic $\left(c_{s} \lesssim v_{g 0}\right)$ and its density perturbation propagates as a hump $\left(\rho_{0}>0\right)$. In both cases we have $v_{g 0}^{\prime}\left(c_{s}-v_{g 0}\right)>0$.

The variation of $v_{g 0}$ with $\omega_{0} / \omega_{c}$ shows that the condition $v_{g 0} \simeq c_{s}$ can be fulfilled in two frequency domains : $\omega_{0} \ll \omega_{c}$ or $\omega_{0} \simeq \omega_{c}$. In the latter case, we get the following approximate expressions for a plasma with $\omega_{p}>\omega_{c}: v_{g 0} \simeq 2 c\left(\omega_{c} / \omega_{p}\right)\left(1-\omega_{0} / \omega_{c}\right)^{1 / 2}, v_{g 0}^{\prime} \simeq$ $-8 c^{2} \omega_{c} / \omega_{p}^{2} /\left(1-\omega_{0} / \omega_{c}\right)$ and $\alpha \chi \simeq 2 \omega_{p} c_{s}\left(1-\omega_{0} / \omega_{c}\right)^{-1 / 2} / c$, where we note that $v_{g 0}^{\prime}<0$; then the condition (60) can be written as

$$
\frac{T_{h}}{T_{h}+T_{i}}\left(1-\frac{v_{T}^{2}}{c^{2}}\left(1-\frac{\omega_{0}}{\omega_{c}}\right)\right)+3>0
$$

which is always true : solitons of the KdV equation exist for sonic whistlers with frequencies near $\omega_{c}$. Moreover their electric field amplitude is given by

$$
\frac{\left|E_{s}\right|^{2}}{16 \pi n_{0} m_{i} c_{s}^{2}} \simeq 8 \frac{\left(c_{s}-v_{g 0}\right)^{2}}{c_{s}^{2}} \frac{c}{c_{s}} \frac{\omega_{c}^{3}}{\omega_{p}^{3}} \frac{\left(1-\omega_{0} / \omega_{c}\right)^{5 / 2}}{\lambda_{0}^{2} / \lambda_{h}^{2}+3},
$$

with the corresponding width and density dip

$$
\frac{c^{2}}{\omega_{c}^{2} l_{s}^{2}} \simeq-\frac{\left(c_{s}-v_{g 0}\right)}{6 c} \frac{\omega_{p}^{3}}{\omega_{c}^{3}} \frac{\left(1-\omega_{0} / \omega_{c}\right)^{1 / 2}}{\lambda_{0}^{2} / \lambda_{h}^{2}+3}, \rho_{0} \simeq-\frac{4\left(c_{s}-v_{g 0}\right)}{c_{s}\left(\lambda_{0}^{2} / \lambda_{h}^{2}+3\right)} .
$$


The soliton is slightly supersonic and the density $\rho$ propagates as a hump. No subsonic soliton exists in this case.

\section{CONCLUSION}

A self-consistent Hamiltonian model based on equations describing the coupled dynamics of electromagnetic whistlers and lower frequency waves in inhomogeneous plasmas is built. Based on this model, the paper extends and generalizes the existing studies by enlarging the domain of their parameters' validity and by providing detailed numerical simulations controlled by the model's invariants and involving various weakly inhomogeneous plasma density structures. Different aspects of whistler turbulence are studied, concerning mainly the development of modulational instabilities and the dynamics of envelope solitons in irregular plasmas.

Modulational instability can develop for supersonic or subsonic whistlers, if the derivative $v_{g 0}^{\prime}=d v_{g 0} / d k$ of the group velocity $v_{g 0}$ is negative or positive, respectively. Simple analytical expressions of the growth rates are provided for the supersonic, subsonic as well as sonic cases. Moreover numerical simulations show that modulational instability can lead to the generation of a beating of stable nonlinear whistlers propagating with a speed near the group velocity.

The whistler envelope soliton has been determined analytically by using the developed model and its propagation in plasmas presenting random density fluctuations and irregular density structures of different scales and amplitudes has been studied, showing that the envelope is very weakly affected by these inhomogeneities, even if the wavelengths and the amplitudes of the phase oscillations inside the envelopes strongly vary. As a linear wave, the whistler soliton can accelerate or decelerate when crossing density gradients, these effects remaining however very weak. Moreover, simulations show for the first time that two whistler solitons colliding one with the other remain unchanged after this collision, independently of their initial amplitudes and velocities.

The propagation of sonic whistler solitons is actually different from that of subsonic and supersonic ones. Their lower frequency perturbation is governed by a KdV-type equation where dispersive effects are however weak. But they are compensated by ponderomotive effects due to the whistler fields which counterbalance the steepening and the wave breaking processes due to the nonlinearity.

\section{ACKNOWLEDGMENTS}

This work was granted access to the HPC resources of IDRIS under the allocation 2017A0010510106 made by GENCI. This work has been done within the LABEX Plas@par project, and received financial state aid managed by the Agence Nationale de la Recherche, 
as part of the programme "Investissements d'avenir" under the reference ANR-11-IDEX0004-02. C.K. acknowledges the "Programme National Soleil Terre" (PNST) and the Centre National d'Etudes Spatiales (CNES, France). The work was carried out with the financial support of the Russian Foundation for Basic Research, project No. 16-52-16010 NTSNIL_a.

\section{REFERENCES}

1

${ }^{1}$ H. J. Beinroth and F. M. Neubauer, Properties of whistler mode waves between 0.3 and $1.0 \mathrm{au}$ from Helios observations, J. Geophys. Res.: Space Phys. 86(A9),7755-7760 (1981). doi:10.1029/JA086iA09p07755.

${ }^{2}$ G. Thejappa and R.J. MacDowall, Evidence for strong and weak turbulence processes in the source region of a local type III radio burst, Astrophys. J. 498,465-478 (1998).

${ }^{3}$ O. Moullard, D. Burgess, C. Salem, A. Mangeney, D. E. Larson, and S. D. Bale. Whistler waves, Langmuir waves and single loss cone electron distributions inside a magnetic cloud: Observations, J. Geophys. Res.: Space Phys. 106(A5),8301-8313 (2001). doi: 10.1029/2000JA900144.

${ }^{4}$ C. Vocks, and G. Mann, Whistler wave excitation by relativistic electrons in coronal loops during solar flares, Astron. Astrophys. 452(1),331-337 (2006). doi:10.1051/00046361:20054042.

${ }^{5}$ R. M. Thorne and B. T. Tsurutani, The generation mechanism for magnetosheath lion roars, Nature 293,384 (1981).

${ }^{6}$ H. K. Wong and C. W. Smith, Electron beam excitation of upstream waves in the whistler mode frequency range, J. Geophys. Res.: Space Phys. 99(A7),13373-13387 (1994). doi: 10.1029/94JA00821.

${ }^{7}$ Y. Zhang, H. Matsumoto, and H. Kojima, Bursts of whistler mode waves in the upstream of the bow shock: Geotail observations, J. Geophys. Res.: Space Phys. 103(A9),2052920540 (1998). doi:10.1029/98JA01371.

${ }^{8}$ F. V. Coroniti, C. F. Kennel, F. L. Scarf, and E. J. Smith, Whistler mode turbulence in the disturbed solar wind, J. Geophys. Res.: Space Phys. 87(A8),6029-6044 (1982). doi:10.1029/JA087iA08p06029.

${ }^{9}$ S. P. Gary and W. C. Feldman, Solar wind heat flux regulation by the whistler instability, J. Geophys. Res.: Space Phys. 82(7),1087-1094 (1977). doi:10.1029/JA082i007p01087.

${ }^{10}$ C. Lacombe, O. Alexandrova, L. Matteini, O. Santoli-k, N. Cornilleau-Wehrlin, A. Mangeney, Y. de Conchy, and M. Maksimovic, Whistler mode waves and the electron heat flux in the solar wind: Cluster observations, Astrophys. J. 796(1),5, (2014).

${ }^{11}$ Y. Omura and H. Matsumoto, Competing processe of whistler and electrostatic instabilities in the magnetosphere, J. Geophys. Res.: Space Phys. 92(A8),8649-8659 (1987). doi: 10.1029/JA092iA08p08649. 
${ }^{12}$ T. F. Bell and R. A. Helliwell, Pulsation phenomena observed in long-duration VLF whistler-mode signals, J. Geophys. Res.: Space Phys. 76(34),8414-8419 (1971). doi: 10.1029/JA076i034p08414.

${ }^{13}$ A. Breneman, C. Cattell, S. Schreiner, K. Kersten, L. B. Wilson, P. Kellogg, K. Goetz, and L. K. Jian, Observations of large-amplitude, narrowband whistlers at stream interaction regions, J. Geophys. Res.: Space Phys. 115(A8),A08104 (2010). doi:10.1029/2009JA014920.

${ }^{14}$ C. T. Russell, D. D. Childers, and P. J. Coleman, Ogo 5 observations of upstream waves in the interplanetary medium: Discrete wave packets, J. Geophys. Res.: Space Phys. 76 (4),845-861 (1971). doi:10.1029/JA076i004p00845.

${ }^{15}$ Guang-Li Huang, De-Yu Wang, and Qi-Wu Song, Whistler waves in Freja observations, J. Geophys. Res.: Space Phys. 109(A2),A02307 (2004). doi:10.1029/2003JA010137..

${ }^{16}$ O. Moullard, A. Masson, H. Laakso, M. Parrot, P. Décréau, O. Santolik, and M. Andre, Density modulated whistler mode emissions observed near the plasmapause, Geophys. Res. Lett. 29(20),361-364 (2002). doi:10.1029/2002GL015101.

${ }^{17}$ R. Pottelette, R. E. Ergun, R. A. Treumann, M. Berthomier, C. W. Carlson, J. P. McFadden, and I. Roth, Modulated electron-acoustic waves in auroral density cavities: Fast observations, Geophys. Res. Lett. 26(16),2629-2632 (1999). doi:10.1029/1999GL900462.

${ }^{18}$ Wang De-Yu and G P Chernov, Envelope soliton in solar radio emission, Chin. Phys. Lett. 17(5),355 (2000).

${ }^{19}$ V.E. Zakharov and E. A. Kuznetsov, Hamiltonian formalism for nonlinear waves, Phys. Usp. 40(11),1087-1116 (1997).

${ }^{20}$ G.W. Kentwell and D.A. Jones, The time-dependent ponderomotive force, Phys. Rep., 145(6), 319-403 (1987).

${ }^{21}$ V. I. Karpman, F. R. Hansen, T. Huld, J. P. Lynov, H. L. Pécseli, and J. Juul Rasmussen, Nonlinear evolution of the modulational instability of whistler waves, Phys. Rev. Lett. 64 (8),890-893 (1990).

${ }^{22}$ B. Eliasson and P. K. Shukla, Theoretical and numerical studies of density modulated whistlers, Geophys. Res. Lett. 31(17),L17802 (2004). doi:10.1029/2004GL020605.

${ }^{23}$ I. Kourakis, P. K. Shukla, and G. E. Morfill, Dynamics of nonlinearly coupled magnetic-field-aligned electromagnetic electron-cyclotron waves near the zero-groupdispersion point in magnetized plasmas, Phys. Plasmas 12(8),082303 (2005). doi: http://dx.doi.org/10.1063/1.1994747.

${ }^{24}$ C. Krafft, A. S. Volokitin, and V. V. Krasnoselskikh, Interaction of energetic particles with waves in strongly inhomogeneous solar wind plasmas, Astrophys. J. 778,111 (2013). doi:10.1088/0004-637X/778/2/111.

${ }^{25}$ C. Krafft, A. S. Volokitin, and V. V. Krasnoselskikh, Langmuir wave decay in inhomogeneous solar wind plasmas: Simulation results, Astrophys. J. 809(2),176 (2015).

${ }^{26}$ C. Krafft, A.S. Volokitin, V.V. Krasnoselskikh, and T. Dudok de Wit, Waveforms of Langmuir turbulence in inhomogeneous solar wind plasmas, J. Geophys. Res.: Space Phys. 119,9369-9382 (2014). doi:10.1002/2014JA020329. 
${ }^{27}$ A. Voshchepynets, A. Volokitin, V. Krasnoselskikh, and C. Krafft, Statistics of electric fields' amplitudes in Langmuir turbulence: A numerical simulation study, J. Geophys. Res.: Space Phys. 122(4),3915-3934 (2017). doi:10.1002/2017JA023898.

${ }^{28} \mathrm{H}$. Washimi, Self-focusing of transverse waves in a magnetoplasma, J. Phys. Soc. Jpn. 34, 1373-1383 (1973). doi:http://dx.doi.org/10.1143/JPSJ.34.1373.

${ }^{29} \mathrm{H}$. Washimi and V. I. Karpman, The ponderomotive force of a high-frequency electromagnetic field in a dispersive medium, JETP 71,1010-1016 (1976).

${ }^{30}$ D.W. Forslund, J.M. Kindel, and E.L. Lindman, Parametric excitation of electromagnetic waves, Phys. Rev. Lett. 29,249-252 (1972).

${ }^{31}$ G.M. Zaslavskii and N.N. Filonenko, Instability of nonlinear periodic waves in a dispersive medium, JETP 29(3),572 (1969).

${ }^{32} \mathrm{C}$. Krafft and A.Volokitin, Interaction of whistler envelope solitons with non-relativistic electron beams in plasmas with density inhomogeneities, Phys. Plasmas, in press, 2018.

\section{APPENDIX A : MODEL'S EQUATIONS}

\section{A. Equation for the whistler field envelope}

Using (1) we can write that $\partial \mathbf{B} / \partial t=\left(\partial / \partial t-i \omega_{0}\right) B e^{-i \omega_{0} t+i k_{0} z}(\mathbf{x}+i \mathbf{y}) / 2+$ c.c. Taking into account that the envelopes are varying slowly in time and space, we obtain that $\partial^{2} \mathbf{B} / \partial t^{2} \simeq\left(-2 i \omega_{0} \partial / \partial t-\omega_{0}^{2}\right) B e^{-i \omega_{0} t+i k_{0} z}(\mathbf{x}+i \mathbf{y}) / 2+$ c.c. and $\nabla^{2} \mathbf{B} \simeq\left(2 i k_{0} \partial / \partial z-k_{0}^{2}\right) B$ $e^{-i \omega_{0} t+i k_{0} z}(\mathbf{x}+i \mathbf{y}) / 2+$ c.c. So we get from (4) that

$$
\frac{1}{c^{2}}\left(-2 i \omega_{0} \frac{\partial B}{\partial t}-\omega_{0}^{2} B\right)-\left(2 i k_{0} \frac{\partial B}{\partial z}-k_{0}^{2} B\right)=\frac{4 \pi}{c}\left(\nabla \times \mathbf{j}_{L}+\nabla \times \mathbf{j}_{e x t}\right),
$$

where $\mathbf{j}_{L}$ is the linear current density corresponding to the plasma (nonresonant) particles, which can be calculated using the Ohm' law as $\mathbf{j}_{L}=-(i \omega / 4 \pi)(\hat{\varepsilon}-\widehat{I}) \mathbf{E} ; \hat{\varepsilon}$ is the cold dielectric tensor with the non vanishing elements $\varepsilon_{x x}=\varepsilon_{y y}=\varepsilon_{\perp}, \varepsilon_{z z}=1-\omega_{p}^{2} / \omega^{2}$ and $\varepsilon_{x y}=$ $-\varepsilon_{y x}=i q ; \widehat{I}$ is the unity tensor. Then Maxwell equations lead to

$$
\nabla \times \mathbf{j}_{L} \simeq \frac{i \omega}{4 \pi c}(\hat{\varepsilon}-\widehat{I}) \frac{\partial \mathbf{B}}{\partial t} \simeq \frac{\omega^{2}}{8 \pi c}\left(\frac{c^{2} k^{2}}{\omega^{2}}-\frac{\mathcal{D}(\omega, k)}{\omega^{2}}-1\right) B e^{-i \omega_{0} t+i k_{0} z}(\mathbf{x}+i \mathbf{y})+\text { c.c. }
$$

When no external current density is included $\left(\mathbf{j}_{e x t}=0\right)$, we get from (A1) that $\mathcal{D}(\omega, k)$ $B \simeq 0$, where $\mathcal{D}(\omega, k)$ can be presented in the form of a differential operator (5). Note that, obviously, order two terms of $\mathcal{D}(\omega, k)$ in the small nonlinear terms including $\rho, \delta v_{e}$ and $\delta B_{z}$ are neglected. Therefore we can write the equation for the magnetic field envelope as

$$
\begin{gathered}
i\left(\frac{\partial \mathcal{D}}{\partial \omega}\right)_{0} \frac{\partial B}{\partial t}-i\left(\frac{\partial \mathcal{D}}{\partial k}\right)_{0} \frac{\partial B}{\partial z}-\frac{1}{2}\left(\left(\frac{\partial^{2} \mathcal{D}}{\partial \omega^{2}}\right)_{0} \frac{\partial^{2} B}{\partial t^{2}}-2\left(\frac{\partial^{2} \mathcal{D}}{\partial k \partial \omega}\right)_{0} \frac{\partial^{2} B}{\partial z \partial t}+\left(\frac{\partial^{2} \mathcal{D}}{\partial k^{2}}\right)_{0} \frac{\partial^{2} B}{\partial z^{2}}\right) \\
+\rho\left(\frac{\partial \mathcal{D}}{\partial \rho}\right)_{0} B+\delta v_{e}\left(\frac{\partial \mathcal{D}}{\partial \delta v_{e}}\right)_{0} B+\frac{\delta B_{z}}{B_{0}}\left(\frac{\partial \mathcal{D}}{\partial\left(\delta B_{z} / B_{0}\right)}\right)_{0} B \simeq 0 .
\end{gathered}
$$


Dividing this expression by $(\partial \mathcal{D} / \partial \omega)_{0}$ and noting that $v_{g 0}=(d \omega / d k)_{0}=-(\partial \mathcal{D} / \partial k)_{0} /(\partial \mathcal{D} / \partial \omega)_{0}$ as well as

$$
v_{g 0}^{\prime}=\frac{\partial v_{g 0}}{\partial k}+v_{g 0} \frac{\partial v_{g 0}}{\partial \omega} \simeq-\left(\left(\frac{\partial^{2} \mathcal{D}}{\partial k^{2}}\right)_{0}+2 v_{g 0} \frac{\partial^{2} \mathcal{D}}{\partial k \partial \omega}+v_{g 0}^{2}\left(\frac{\partial^{2} \mathcal{D}}{\partial \omega^{2}}\right)_{0}\right) /\left(\frac{\partial \mathcal{D}}{\partial \omega}\right)_{0}
$$

and

$$
\left(\frac{\partial^{2} \mathcal{D}}{\partial \omega^{2}}\right)_{0} \frac{\partial^{2} B}{\partial t^{2}}-2\left(\frac{\partial^{2} \mathcal{D}}{\partial k \partial \omega}\right)_{0} \frac{\partial^{2} B}{\partial z \partial t}+\left(\frac{\partial^{2} \mathcal{D}}{\partial k^{2}}\right)_{0} \frac{\partial^{2} B}{\partial z^{2}} \simeq\left(\left(\frac{\partial^{2} \mathcal{D}}{\partial k^{2}}\right)_{0}+2 v_{g 0}\left(\frac{\partial^{2} \mathcal{D}}{\partial k \partial \omega}\right)+v_{g 0}^{2}\left(\frac{\partial^{2} \mathcal{D}}{\partial \omega^{2}}\right)_{0}\right) \frac{\partial^{2} B}{\partial z^{2}}
$$

we get finally (6), observing that

$$
\mathcal{D}(\omega, k) \simeq k^{2} c^{2}-\omega^{2}+\frac{\omega_{p}^{2}(1+\rho) \omega}{\omega-k \delta v_{e}-\omega_{c}\left(1+\delta B_{z} / B_{0}\right)} .
$$

\section{B. Hamiltonian of the system}

In order to obtain the Hamiltonian (18) of the system of equations (12)-(13)-(17), let us multiply (17) by $\partial \mathcal{B}^{*} / \partial t$, add the complex conjugate and integrate over the plasma size $L$. The first term is obviously vanishing. The second term can be expressed as follows, using integration by parts and the fact that the field and its derivatives are vanishing at infinity

$$
i v_{g 0} \int \frac{d z}{L}\left(\frac{\partial \mathcal{B}}{\partial z} \frac{\partial \mathcal{B}^{*}}{\partial t}-\frac{\partial \mathcal{B}^{*}}{\partial z} \frac{\partial \mathcal{B}}{\partial t}\right)=-\frac{i v_{g 0}}{2} \frac{\partial}{\partial t} \int \frac{d z}{L}\left(\mathcal{B} \frac{\partial \mathcal{B}^{*}}{\partial z}-\mathcal{B}^{*} \frac{\partial \mathcal{B}}{\partial z}\right)
$$

For the same reasons, the third term can be written as

$$
\frac{v_{g 0}^{\prime}}{2} \int \frac{d z}{L}\left(\frac{\partial^{2} \mathcal{B}}{\partial z^{2}} \frac{\partial \mathcal{B}^{*}}{\partial t}+\frac{\partial^{2} \mathcal{B}^{*}}{\partial z^{2}} \frac{\partial \mathcal{B}}{\partial t}\right)=-\frac{v_{g 0}^{\prime}}{2} \frac{\partial}{\partial t} \int \frac{d z}{L}\left|\frac{\partial \mathcal{B}}{\partial z}\right|^{2}
$$

For the fourth term we use the following decomposition

$$
\int \frac{d z}{L}\left(\rho \mathcal{B} \frac{\partial \mathcal{B}^{*}}{\partial t}+\rho \mathcal{B}^{*} \frac{\partial \mathcal{B}}{\partial t}\right)=\int \frac{d z}{L} \rho \frac{\partial}{\partial t}|\mathcal{B}|^{2}=\frac{\partial}{\partial t} \int \frac{d z}{L} \rho|\mathcal{B}|^{2}-\int \frac{d z}{L}|\mathcal{B}|^{2} \frac{\partial \rho}{\partial t} .
$$

In the fifth term we insert the low frequency equations (15)-(16) so that we can write

$$
\int \frac{d z}{L}\left(\frac{\partial \Psi}{\partial z} \mathcal{B} \frac{\partial \mathcal{B}^{*}}{\partial t}+\frac{\partial \Psi}{\partial z} \mathcal{B}^{*} \frac{\partial \mathcal{B}}{\partial t}\right)=\frac{\partial}{\partial t} \int \frac{d z}{L}\left(\frac{\partial \Psi}{\partial z}|\mathcal{B}|^{2}\right)-\int \frac{d z}{L}|\mathcal{B}|^{2} \frac{\partial^{2} \Psi}{\partial t \partial z}
$$

Then, summing the two last terms of (A4)-(A5), replacing $\partial \rho / \partial t$ using (16) and $\partial^{2} \Psi / \partial t \partial z$ using (15), we get that

$$
\omega_{0} \int \frac{d z}{L}|\mathcal{B}|^{2} \frac{\partial \rho}{\partial t}+\frac{k_{0} \omega_{c}}{\left(\omega_{0}-\omega_{c}\right)} \int \frac{d z}{L}|\mathcal{B}|^{2} \frac{\partial^{2} \Psi}{\partial t \partial z}=-\frac{k_{0}^{2}\left(\omega_{0}-\omega_{c}\right)}{2 \omega_{p}^{2}} \frac{\partial}{\partial t} \int \frac{d z}{L}\left(\left(\frac{\partial \Psi}{\partial z}\right)^{2}+c_{s}^{2} \rho^{2}\right) .
$$


Finally, for the sixth term we can write that

$$
\int \frac{d z}{L}|\mathcal{B}|^{2}\left(\mathcal{B} \frac{\partial \mathcal{B}^{*}}{\partial t}+\mathcal{B}^{*} \frac{\partial \mathcal{B}}{\partial t}\right)=\frac{1}{2} \frac{\partial}{\partial t} \int \frac{d z}{L}|\mathcal{B}|^{4} .
$$

Gathering all the terms of (A2)-(A7) leads to the Hamiltonian

$$
\begin{aligned}
\mathcal{H}= & \int \frac{d z}{L}\left(\frac{i v_{g 0}}{2}\left(\mathcal{B} \frac{\partial \mathcal{B}^{*}}{\partial z}-\mathcal{B}^{*} \frac{\partial \mathcal{B}}{\partial z}\right)+\frac{v_{g 0}^{\prime}}{2}\left|\frac{\partial \mathcal{B}}{\partial z}\right|^{2}+\frac{\omega_{p}^{2} \omega_{0} \rho|\mathcal{B}|^{2}}{\left(\omega_{c}-\omega_{0}\right) \mathcal{D}_{0}^{\prime}}\right. \\
& \left.-\frac{k_{0}^{2}}{2 \mathcal{D}_{0}^{\prime}}\left(\left(\frac{\partial \Psi}{\partial z}+\frac{\omega_{c} \omega_{p}^{2}}{k_{0}\left(\omega_{c}-\omega_{0}\right)^{2}}|\mathcal{B}|^{2}\right)^{2}+c_{s}^{2} \rho^{2}\right)\right),
\end{aligned}
$$

which is similar to (18) if one takes into account (14).

\section{APPENDIX B : NON STATIONARY PART OF THE PONDEROMOTIVE FORCE}

Let us calculate the non stationary part of the ponderomotive force. The slowly varying parallel fluid motion can be written as

$$
m_{e} \frac{\partial v_{z}}{\partial t}+m_{e} v_{z} \frac{\partial}{\partial z} v_{z}+e E_{z}=-\frac{e}{c}\left\langle\left(\mathbf{v}_{\perp} \times \mathbf{B}_{\perp}\right)_{z}\right\rangle=\frac{\langle F\rangle}{n_{0}} \simeq \frac{1}{n_{0} c}\left\langle j_{x} B_{y}-j_{y} B_{x}\right\rangle,
$$

where $E_{z}$ is the low frequency part of the parallel electric field and $\langle F\rangle$ is the ponderomotive force per unit volume. At the first order, we can write the Newton equations for the electrons in the perpendicular plane in the form

$$
\frac{d v_{x}}{d t}=-\frac{e}{m_{e}} E_{x}-\frac{e}{m_{e} c} v_{y} B_{0}, \quad \frac{d v_{y}}{d t}=-\frac{e}{m_{e}} E_{y}+\frac{e}{m_{e} c} v_{x} B_{0} .
$$

Let us define $v^{+}=v_{x}+i v_{y}, v^{-}=v_{x}-i v_{y}, E^{+}=E_{x}+i E_{y}$ and $E^{-}=E_{x}-i E_{y}$, which provide the motion equations in the form

$$
\frac{d v^{ \pm}}{d t}=-\frac{e}{m_{e}} E^{ \pm} \pm i \omega_{c} v^{ \pm}
$$

Searchig solutions proportional to $e^{-i \omega_{0} t}$ we get the velocity perturbations as a function of the electric field perturbation

$$
\delta v^{ \pm}=-\frac{e}{m_{e}\left(\omega_{0} \pm \omega_{c}\right)^{2}} \frac{\partial \delta E^{ \pm}}{\partial t} .
$$

Keeping only the field $E^{-}$, as for whistlers we have $E_{x}=-i E_{y}$, i.e. $E^{+}=0$, we get the real values of the velocities

$$
\left(\operatorname{Re} v_{x}, \operatorname{Re} v_{y}\right)=\frac{e}{2 m_{e}} \frac{1}{\omega_{0}-\omega_{c}}\left(\operatorname{Im} E^{-}, \operatorname{Re} E^{-}\right),
$$




$$
\left(\operatorname{Re} \delta v_{x}, \operatorname{Re} \delta v_{y}\right)=\frac{e}{2 m_{e}\left(\omega_{0}-\omega_{c}\right)^{2}} \frac{\partial}{\partial t}\left(-\operatorname{Re} \delta E^{-}, \operatorname{Im} \delta E^{-}\right) .
$$

For the fields we get similarly the first order perturbations

$$
\operatorname{Re} \delta B_{x} \simeq-\frac{k_{0} c}{\omega_{0}} \frac{\partial \operatorname{Im} \delta E_{y}}{\omega_{0} \partial t}, \quad \operatorname{Re} \delta B_{y} \simeq \frac{k_{0} c}{\omega_{0}} \frac{\partial \operatorname{Im} \delta E_{x}}{\omega_{0} \partial t} .
$$

The above equations allow us to calculate the ponderomotive force's perturbation

$$
\frac{\delta F}{n_{0}}=-\frac{e}{c}\left[\operatorname{Re} v_{x} \operatorname{Re} \delta B_{y}-\operatorname{Re} v_{y} \operatorname{Re} \delta B_{x}+\operatorname{Re} \delta v_{x} \operatorname{Re} B_{y}-\operatorname{Re} \delta v_{y} \operatorname{Re} B_{x}\right]
$$

so that

$$
\frac{\delta F}{n_{0}}=\frac{1}{8 \pi} \frac{\omega_{p}^{2} \omega_{c}}{\omega_{0}\left(\omega_{0}-\omega_{c}\right)^{2}} \frac{k}{\omega_{0}} \frac{\partial}{\partial t}\left|\delta E_{x}\right|^{2} .
$$

Averaging on time, we get the non stationary part of the ponderomotive force as

$$
\frac{\langle F\rangle}{n_{0}}=\frac{\omega_{p}^{2} \omega_{c}}{\omega_{0}\left(\omega_{0}-\omega_{c}\right)^{2}} \frac{k}{\omega_{0}} \frac{\partial}{\partial t} \frac{\left\langle|E|^{2}\right\rangle}{16 \pi} .
$$

\section{APPENDIX C : NORMALIZED MODEL'S EQUATIONS AND NUMERICAL SCHEME}

Figures are presented using dimensionless variables. The time $t$, the distance $z$ and the whistler magnetic field $B$ are normalized by the electron gyrofrequency $\omega_{c}$, by $\omega_{c} / c$ and by the modulus $B_{0}$ of the ambient magnetic field, respectively. All velocities, as the group velocity $v_{g 0}$, the ion acoustic velocity $c_{s}$ and the electron thermal velocity $v_{T}$ are normalized by the light velocity $c$, except of the slow velocity $V$ of the electrons which is normalized by $c_{s}$. The normalized forms of the equations (6), (12) and (13) are (the same name is given to the normalized variables used below, and to the non normalized ones used in the body of the text)

$$
\begin{gathered}
i \frac{\partial B}{\partial t}+i v_{g 0} \frac{\partial B}{\partial z}+\frac{v_{g 0}^{\prime}}{2} \frac{\partial^{2} B}{\partial z^{2}}=-\frac{\omega_{0} \omega_{p}^{2}}{\omega_{c}\left(\omega_{0}-\omega_{c}\right) \mathcal{D}_{0}^{\prime}}\left(\rho+\frac{\omega_{c}}{\omega_{0}-\omega_{c}} c_{s} \frac{k_{0} V}{\omega_{0}}\right) B, \\
\frac{\partial}{\partial t}\left(c_{s} V-\frac{\omega_{c}^{3}}{\left(\omega_{0}-\omega_{c}\right)^{2}} \frac{m_{e}}{m_{i}} \frac{|B|^{2}}{4 k_{0}}\right)+\frac{\partial}{\partial z}\left(c_{s}^{2} \rho+\frac{m_{e}}{m_{i}} \frac{\omega_{0} \omega_{c}^{2}}{\left(\omega_{0}-\omega_{c}\right)} \frac{|B|^{2}}{4 k_{0}^{2}}\right) \simeq 0, \\
\frac{\partial \rho}{\partial t}+c_{s} \frac{\partial V}{\partial z} \simeq 0,
\end{gathered}
$$

where $\omega_{c}=1, \rho=\delta n_{i} / n_{0}$ and $V=\delta v_{e} / c_{s}$. The corresponding numerical scheme used for integration of the equations is given by

$$
B_{\kappa}^{n+1}=\frac{\left(1-i \tau \Omega_{\kappa} / 2\right)}{\left(1+i \tau \Omega_{\kappa} / 2\right)} B_{\kappa}^{n}+
$$




$$
\begin{gathered}
+\frac{i \tau}{\left(1+i \tau \Omega_{\kappa} / 2\right)} \frac{\omega_{0} \omega_{p}^{2}}{2 \mathcal{D}_{0}^{\prime}\left(\omega_{0}-\omega_{c}\right)}\left[(\rho B)_{\kappa}^{n+1}+(\rho B)_{\kappa}^{n}+\frac{\omega_{c}}{\omega_{0}-\omega_{c}} \frac{c_{s} k_{0}}{\omega_{0}}\left((V B)_{\kappa}^{n+1}+(V B)_{\kappa}^{n}\right)\right] \\
\rho_{\kappa}^{n+1}=\rho_{\kappa}^{n}-\frac{i \tau \kappa c_{s}}{2}\left(V_{\kappa}^{n+1}+V_{\kappa}^{n}\right) \\
V_{\kappa}^{n+1}=\frac{\left(1-\tau^{2} \kappa^{2} c_{s} / 4\right)}{\left(1+\tau^{2} \kappa^{2} c_{s} / 4\right)} V_{\kappa}^{n}+\frac{-i \tau \kappa c_{s} \rho_{\kappa}^{n}}{\left(1+\tau^{2} \kappa^{2} c_{s} / 4\right)}+ \\
+\frac{m_{e}}{\left(1+\tau^{2} \kappa^{2} c_{s} / 4\right) k_{0} c_{s}\left(\omega_{0}-\omega_{c}\right)}\left(\frac{\left(|B|^{2}\right)_{\kappa}^{n+1}-\left(|B|^{2}\right)_{\kappa}^{n}}{\left(\omega_{0}-\omega_{c}\right)}+i \tau \kappa \frac{\omega_{0}}{2 k_{0}}\left(\left(|B|^{2}\right)_{\kappa}^{n+1}+\left(|B|^{2}\right)_{\kappa}^{n}\right)\right)
\end{gathered}
$$

where $\Omega_{\kappa}=v_{g 0} \kappa+v_{g 0}^{\prime} \kappa^{2} / 2$ and $\epsilon=\tau^{2} \kappa^{2} c_{s} / 4 ; \tau$ is the time step ; $\rho_{\kappa}^{n}, V_{\kappa}^{n}, B_{\kappa}^{n}$ are the Fourier components at time $n$ of the density, the fluid velocity and the magnetic field envelope. 